\title{
ATOMISTIC MODELING OF SURFACE AND BULK PROPERTIES OF Cu, Pd AND THE Cu-Pd SYSTEM
}

\author{
Guillermo Bozzolo $^{\mathrm{a}, \mathrm{b},{ }^{*}}$, Jorge E. Garcés ${ }^{\mathrm{a}, \mathrm{c}}$, Ronald D. Noebe ${ }^{\mathrm{b}}$, Phillip Abel ${ }^{\mathrm{b}}$ \\ and Hugo O. Mosca ${ }^{d}$
}

${ }^{a}$ Ohio Aerospace Institute, 22800 Cedar Point Rd., Cleveland, OH 44142, USA

b NASA Glenn Research Center, Cleveland, OH 44135, USA

${ }^{c}$ Centro Atómico Bariloche, 8400 Bariloche, Argentina

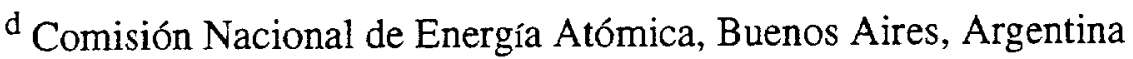

\begin{abstract}
The BFS method for alloys is applied to the study of the Cu-Pd system. A variety of issues are analyzed and discussed, including the properties of pure $\mathrm{Cu}$ or Pd crystals (surface energies, surface relaxations), $\mathrm{Pd} / \mathrm{Cu}$ and $\mathrm{Cu} / \mathrm{Pd}$ surface alloys, segregation of $\mathrm{Pd}$ (or $\mathrm{Cu}$ ) in $\mathrm{Cu}$ (or $\mathrm{Pd}$ ), concentration dependence of the lattice parameter of the high temperature fcc CuPd solid solution, the formation and properties of low temperature ordered phases, and order-disorder transition temperatures. Emphasis is made on the ability of the method to describe these properties on the basis of a minimum set of BFS universal parameters that uniquely characterize the $\mathrm{Cu}-\mathrm{Pd}$ system.
\end{abstract}

PACS: 61.43.Bn, 61.66.Dk, 61.72.Bb, 64.60.Cn, 64.75.+g, 68.35.-p, 68.55.-a

Keywords: Copper, Palladium, Surface energies, Segregation, Surface relaxation, Surface alloys, Alloy surfaces, Solid solution, Order-disorder transitions, Computational Methods.

\section{INTRODUCTION}

In the last few years, there has been an increasing demand for precisely designed materials with very specific properties. The current method for developing new materials, based on a painstaking empirical approach, is both time-consuming and extremely costly. While the virtual design of new

* Corresponding author: Guillermo H. Bozzolo, NASA Glenn Research Center, Mail Stop 23-2, Cleveland, OH 44135, USA. Phone: +1 (216) 433-5824, FAX: +1 (216) 433-5170

(Guillermo.H.Bozzolo@grc.nasa.gov)

his report is a preprint of an article submitted to journal for publication. Because of changes that lay be made before formal publication, this reprint is made available with the understanding 
materials through complex computer simulations is still many years away, they already play a significant role in materials design, and offer several advantages over a strictly laboratory style development program. Their success depends on the availability of a unified approach that provides the same level of simplicity and accuracy for any possible application, whether it is directed to surface and/or bulk analysis. The trend to incorporate atomistic simulations as a standard tool in the analysis of complex systems has imposed high expectations on the range of applicability of such methods, their computational efficiency, their ease of implementation, and the type of output that they provide. While it seems possible that no new approach will be widely accepted until it is shown to work reliably in the broadest sense, it is important to understand that the starting point is, in itself, the strongest requirement imposed: materials design is a very complicated issue involving many variables, including time, temperature, pressure, and all the possible combinations of elements in the periodic table [1].

With these requirements in mind, efficient quantum approximate methods (QAM's) [2-6] have been developed, each offering a fresh outlook on important issues commonly unavailable experimentally or too demanding, computationally, for accurate first-principles methods. Almost independently of their foundation and formulation, QAM's rely on simplifications which, as a result, inevitably require the introduction of parameters. This is a common feature to all methods, besides any other assumption on the mathematical expressions for potentials or any other function that might appear in the theory as a result of an approximation. Additional restrictions generally apply, resulting in limitations on the efficiency or accuracy of the method in terms of type of lattice structure, number and type of element. More important than these common restrictions, one basic limitation shared by all these methods consists of the ability of these parameters to reproduce physical properties of the system at hand regardless of the particular feature under study. For example, it is not unusual that most methods rely on parameters derived from experimentally determined bulk properties. While this fact alone is not a limitation, it becomes one when the underlying formalism does not support the use of these same parameters for the study of, for example, surface problems. In some other cases, the parameters have a low level of transferability, in the sense that parameters determined for a specific element from a specific source might require an adjustment when that same element is in a different chemical or structural environment. New fitting or adjustment is then required, thus particularizing the resulting parameters for the one specific application at hand. As a result, and in order to keep the delicate but necessary balance 
between accuracy and efficiency, different methods are therefore generally used to deal with bulk crystals, surfaces or interfaces, defects, temperature effects, etc., or, as is sometimes the case, changes in the formulation of a given method are needed when dealing with one type of problem or another are made. One way to solve the problem of transferability is to develop the operational equations of the methods, and the underlying concepts from which they are derived, in a way such that the parameters enter these equations without any particular dependence on any specific situation, whether it involves structural or chemical defects. To that effect, a new QAM, the BFS method for alloys [4], was recently introduced, providing an alternative approach for performing atomistic simulations, by overcoming most of the limitations mentioned above, as nothing in its formulation imposes restrictions on the number and type of elements or the structure under study.

The BFS method overcomes these difficuities simply because of its interpretation and modeling of the alloy formation process [4]. While a mathematical description of the method will be given in the next section, in this section we concentrate on the underlying concepts and their meaning. In BFS, any given system, regardless of its composition and structure is always modeled in terms of two independent virtual processes which, properly coupled, are meant to result in the final state that is being studied. One of these virtual processes relates to the structural changes in the environment of any given atom (strain), and it consists of defining, for every atom, a virtual (perfect) monatomic equivalent crystal of its own species in a state of isotropic compression or expansion with respect to equilibrium. The amount of compression or expansion is defined so that the electron density in the vicinity of the atom in the real crystal resembles, in average, the corresponding one in the expanded or compressed perfect equivalent crystal [5]. The availability of a universal binding energy relationship (UBER) [5,7] to describe such isotropic transformations ensures an accurate and energetically correct description of the process, particularly for small departures from equilibrium. The other virtual process accounts for changes in the chemical environment of that same atom (chemical). Once again, another virtual perfect crystal, in a state of isotropic compression or expansion, is assigned. To decouple structural and chemical effects, it is necessary to introduce additional restrictions in the definition of these processes. In the first case, all atoms surrounding a given reference atom are considered as being of the same atomic species as the reference atom, thus freezing compositional degrees of freedom in the determination of the equivalent crystal associated with structural changes. In the second case, the surrounding atoms retain their chemical identity, but are forced to occupy equilibrium lattice sites of a lattice charac- 
teristic of the reference atom. More details on the nature, characteristics, and modeling of these processes, will be provided in the following section. Here, it is just worth noting that, regardless of the details, both processes share one particular feature: any system is described in the same way, i.e., by means of virtual, ideal bulk crystals. Whether the system involves the presence of surfaces or other extended defects, or a varying chemical composition, etc., the modeling of the formation process is done by means of these virtual crystals which, in their definition (their composition and structure), account for the particular features of the real system that they represent. The environment seen by an atom in the real crystal is translated into an equivalent, perfect crystal. The parameters used in the BFS method describe the virtual processes and the properties of these virtual crystals. As such, they are somewhat blind to the otherwise different systems that they describe, as they are always applied to the energetics of perfect, virtual, crystals. In all cases they describe deviations from an equilibrium state of the equivalent crystal, thus eliminating a direct correlation with the nature of the actual situation which they are meant to represent. For example, the formation of a single vacancy is, in terms of the parameters used, no different from the substitution of a neighboring atom for one of a different species, or a surface defect. Therefore, it is expected that if the theory allows for an unequivocal definition of these virtual crystals, properly endowed with the necessary information on the real crystals that they represent, the parameters will then have the same level of reliability, or be able to extract the same amount and quality of information via the equations of the method, in any kind of situation.

It is also worth noting that the parameterization of the BFS method implies a somewhat different approach for the interaction between different atoms. In general, most approaches deal with this issue by introducing some sort of interaction potential with any parameter describing each constituent remaining unchanged. In BFS, it is precisely the set of parameters describing the pure element what is perturbed in order to account for the distortions introduced by the nearby presence of a different element or defect. In doing so, an additional advantage is thus introduced in the methodology, as the number of parameters is reduced to a minimum and their transferability is therefore guaranteed. It is, however, an additional burden on the method, as too much critical information must then be carried by a very small number of parameters. From a practical standpoint, however, it is obviously a matter of balance between the advantages and disadvantages what ultimately translates into an efficient and accurate method with a maximum range of applicability. 
Meant as a contribution to the process of linking modeling to experimental analysis, the purpose of this work is to illustrate, with a thorough application of BFS to the study of a binary system, the power as well as the limitations of procuring such balance between physical accuracy and flexibility in the spectrum of possible applications. At the same time, this work is meant to provide a general view of the variety of issues that can be tackled with quantum approximate methods. In what follows, we will show that the implementation of a simple and straightforward unified approach to single crystals, alloys, surfaces and bulk properties, is possible and, to a great extent, agreeable with current available experimental evidence.

Taking the binary $\mathrm{Cu}-\mathrm{Pd}$ system as an example, and representing the basic features of the binary systems by means of its phase diagram, this article covers a large number of them, including: 1) application to the analysis of single crystals and their buik and surface properties, including surface energies, surface structure, and multilayer relaxation, 2) identification of the segregating species, 3 ) application to the formation of surface alloys for arbitrary coverage, thin film growth patterns, and interdiffusion, 4) description of ordered phases, their bulk structure, symmetry, defect structure, surface energies and segregation patterns, analysis of order-disorder transitions and determination of critical temperatures, 5) prediction and analysis of metastable structures and their alternative ordering patterns, and 6) description of the physical properties of the solid solution. In a schematic way, Fig. 1 summarizes the applications studied in this work, as well as other applications that can also be modeled, in the framework of the accepted phase diagram for the Cu-Pd binary system.

The paper is organized as follows. Section 2 describes the BFS method for alloys [4], including a brief description of Equivalent Crystal Theory (ECT) [5], the methodology used for the calculation of pure element properties. With the purpose of validating the single element parameters used as input in BFS, Section 3 deals with a brief review of ECT calculations of single element properties, including surface energies, surface structure and relaxation. Section 4 presents the main results of this paper, covering the different aspects of the Cu-Pd system listed above.

\section{THE BFS METHOD}

The BFS method has been applied to a variety of problems, ranging from bulk properties of solid solution fcc alloys [9] and the defect structure in ordered bcc alloys $[10,11]$ to more specific 
applications including detailed studies of the structure and composition of alloy surfaces and surface alloys [12]. These studies provide confidence in the method for application to the problem of site substitution in ordered B2 compounds [13]. In what follows, we provide a brief description of the operational equations of BFS. The reader is encouraged to seek further details in previous papers where a detailed presentation of the foundation of the method, its basis in perturbation theory and a discussion of the approximations made are clearly shown [9-13].

The BFS method provides a simple algorithm for the calculation of the energy of formation $\Delta H$ of an arbitrary alloy (the difference between the energy of the alloy and that of its individual constituents). In BFS, the energy of formation is written as the superposition of elemental contributions of all the atoms in the alloy

$$
\Delta H=\sum_{i} \varepsilon_{i}
$$

For each atom, we partition the energy into two parts: a strain energy, $\varepsilon_{\mathrm{i}}{ }^{\mathrm{S}}$, and a chemical energy, $\varepsilon_{i}{ }^{C}$, contribution. The first specifically relates to the atomic positions of the neighboring atoms to atom $i$, regardless of their chemical identity. For its calculation, we use the actual geometrical distribution of the atoms in the alloy surrounding atom $i$, computed as if all of its neighbors were of the same species as atom $i$. The BFS strain energy differs from the commonly defined strain energy in that the actual chemical environment is replaced by that of a monoatomic crystal. Its calculation is then straightforward, even amenable to first-principles techniques.

The chemical environment of atom $i$ is considered in the computation of the BFS chemical energy contribution, where the surrounding atoms maintain their identity but are forced to occupy equilibrium lattice sites corresponding to the reference atom $i$. A straightforward approach for the calculation of the chemical energy is defined, properly parameterizing the interaction between dissimilar atoms.

Thus defined, the BFS strain and chemical energy contributions take into account different effects, i.e., geometry and composition, computing them as isolated effects. A coupling function, $g_{i}$, restores the relationship between the two terms. This factor is defined in such a way as to properly consider the asymptotic behavior of the chemical energy, where chemical effects are negligi- 
ble for large separations between dissimilar atoms. Summarizing, the contribution to the energy of formation of atom $i$ is then

$\varepsilon_{i}=\varepsilon_{i}^{S}+\bar{g}_{i} \varepsilon_{i}^{C}$

\subsection{CALCULATION OF THE BFS STRAIN ENERGY}

The BFS strain energy can be computed by any method appropriate for the calculation of pure element crystals. As in previous applications of BFS, however, we use Equivalent Crystal Theory (ECT) [5] for its computation, due to its proven ability to provide accurate and computationally economical answers to most general situations. ECT is based on an exact relationship between the total energy and atomic locations and applies to surfaces and defects in both simple and transition metals as in covalent solids. Lattice defects and surface energies are determined via perturbation theory on a fictitious, equivalent single crystal whose lattice parameter is chosen to minimize the perturbation. The energy of the equivalent crystal as a function of its lattice constant, is given by a universal binding energy relationship [7]. ECT is based on the concept that there exists, for each atom $i$, a certain perfect equivalent crystal with its lattice parameter fixed at a value so that the energy of atom $i$ in the equivalent crystal is the BFS strain energy contribution $\varepsilon_{\mathrm{i}}{ }^{\mathrm{S}}$. This equivalent crystal differs from the actual ground state crystal only in that its lattice constant may be different from the ground state value. We compute $\varepsilon_{\mathrm{i}}^{\mathrm{S}}$ via perturbation theory, where the perturbation arises from the difference in the ion core electronic potentials of the actual defect solid and those of the effective bulk single crystal. The simplified perturbation series for $\varepsilon_{i}{ }^{S}$ is of the form [5]

$$
\varepsilon_{i}^{s}=E_{c}\left\{F\left[a_{1}^{*}(i)\right]+\sum_{j} F\left[a_{2}^{*}(i, j)\right]+\sum_{j, k} F\left[a_{3}^{*}(i, j, k)\right]+\sum_{p, q} F\left[a_{4}^{*}(i, p, q)\right]\right\}=\sum_{n=1}^{4} \sigma_{n}
$$

where

$$
F[x]=1-(1+x) e^{-x}
$$


and $E_{c}$ is the cohesive energy of element $i$. Four different contributions to the energy of atom $i$, which find their origin in four different perturbations, are singled out. The linear independence attributed between these four terms is consistent with the limit of small perturbations which is assumed in the formulation of ECT. The first term, $\sigma_{i}=E_{i} F\left[a_{1}^{*}(i)\right]$, contributes when average neighbor distances are altered via defect or surface formation (i.e., changes in coordination). It can be thought as representing local atom density changes, In most cases, this "volume" term is the leading contribution to $\varepsilon_{i}$ and in the case of isotropic volume deformations, it gives $\varepsilon_{\mathrm{i}}{ }^{S}$ to the accuracy of the UBER [7], given by Eq. 4. The higher order terms are relevant for the case of anisotropic deformations [4]. The second term, $\sigma_{2}=E_{c} F\left[a_{2}^{*}(i, j)\right]$, is a two-body term which accounts for the increase in energy when $\mathrm{N}$ bonds are compressed below their equilibrium value. The third term, $\sigma_{3}=E_{c} F\left[a_{3}^{*}(i, j, k)\right]$, accounts for the increase in energy that arises when bond angles deviate from their equilibrium values of the undistorted single crystal, and the fourth term, $\sigma_{4}=E_{c} F\left[a_{4}^{*}(i, p, q)\right]$, describes face diagonal anisotropies (see Ref. 5). In all cases considered in this work, a rigorous application of ECT is reduced to that of its two leading terms, which describe average density contributions and bond-compression anisotropies. We neglect the three- and fourbody terms dealing with the bond angle and face-diagonal anisotropies, except for one single example (surface energy and relaxation of monatomic crystals), where it will be shown that, for metals, their contribution is exceedingly small [5].

We now apply this formalism to the calculation of the BFS strain energy contribution, $\varepsilon_{i}{ }^{S}$, of atom $i$. To do so, the ECT perturbation equation [5] is written in terms of the distances $r_{j}$ between atom $i$ and its NN and NNN,

$N R_{1}^{p_{i}} e^{-\alpha_{i} R_{1}}+M R_{2}^{p_{i}} e^{-\left(\alpha_{i}+\frac{1}{\lambda_{i}}\right) R_{2}}=\sum_{j} r_{j}^{p_{i}} e^{-\left(\alpha_{i}+S\left(r_{j}\right)\right) r_{j}}$

where $N$ and $M$ are the number of NN and NNN respectively, and where $p, l, \alpha$ and $\lambda$ are ECT parameters that describe element $i, r$ denotes the distance between the reference atom and its neighbors, $S(r)$ describes a screening function [4] and the sum runs over all NN and NNN. $R_{1}$ and $R_{2}$ denote the NN and NNN distances in this equivalent crystal. This equation determines the lattice parameter of a perfect equivalent crystal where the reference atom $i$ has the same energy as it has in the geometrical environment of the alloy under study. 
Once the lattice parameter of the (strain) equivalent crystal, $a^{S}$, is determined, the BFS strain energy contribution is computed using the UBER [7], which contains all the relevant information concerning a single-component system:

$$
\varepsilon_{i}^{S}=E_{C}^{i}\left(1-\left(1+a_{i}^{S^{*}}\right) e^{-a_{i}^{s^{*}}}\right)
$$

where the scaled lattice parameter $a_{i}^{S *}$ is given by

$$
a_{i}^{S^{*}}=q \frac{\left(a_{i}^{S}-a_{e}^{i}\right)}{l}
$$

where $q$ is the ratio between the equilibrium Wigner-Seitz radius and the equilibrium lattice parameter $a_{e}{ }^{i}$. When ECT is applied to the study of surfaces of monatomic crystals ( $\mathrm{Cu}$ and $\mathrm{Pd}$ ), all four terms should be included in the calculations. However, when considering rigid surfaces (i.e., no interlayer relaxation) all bond lengths and angles retain their bulk equilibrium values, thus $F\left[a_{2}^{*}\right]=F\left[a_{3}^{*}\right]=F\left[a_{4}^{*}\right]=0$. The rigid surface energy is therefore obtained by solving for the "volume" term represented by $F\left[a_{1}^{*}\right]$ only. If we consider a rigid displacement of the surface layer towards the bulk, as is the case in most metallic surfaces, the higher-order terms become finite: some bonds are compressed, contributing to $F\left[a_{2}^{*}\right]$, the bond angles near the surface are distorted as well as the difference in length between face diagonals in some cases, generating an increase in energy via $F\left[a_{3}^{*}\right]$ and $F\left[a_{4}^{*}\right]$, respectively. For the cases studied in this paper, those additional contributions to $\varepsilon_{i}^{S}$ are generally small, usually representing $1 \%$ to $2 \%$ of the total energy. The corresponding energy contribution $\sigma_{2}$ is directly computed using [14]

$$
\sigma_{2}=E_{C} \sum_{n=1}^{N_{s}} \sum_{m=1}^{M_{n}} \frac{\theta_{m n}}{L_{m n}} F\left(a_{m n}^{*}\right)
$$

where $N_{s}$ is the number of atoms in the solid, $\theta_{m n}=1$ if $a_{m n}^{*} \leq 0$ and $\theta_{m n}=0$ otherwise, $M_{n}$ is the number of NN of atom $n, L_{m n}$ is the number of NN of atom $m$ or $n$, whichever is smaller, and $a_{m n}^{*}$ is given by 
$a_{m n}^{*}=\frac{\frac{R_{m n}}{c_{1}}-r_{W S E}}{l}$

where $R_{m n}$ is the distance between atoms $m$ and $n$, and $\mathrm{c}_{1}$ is the ratio between the equilibrium NN distance and the equilibirum Wigner-Seitz radius.

\subsection{CALCULATION OF THE BFS CHEMICAL ENERGY}

The BFS chemical energy is obtained by a similar procedure. As opposed to the strain energy term, the surrounding atoms retain their chemical identity, but are forced to be in equilibrium lattice sites of an equilibrium (otherwise monoatomic) crystal $i$. The BFS equation for the chemical energy is given by

$$
N R_{1}^{p_{i}} e^{-\alpha_{i} R_{1}}+M R_{2}^{p_{i}} e^{-\left(\alpha_{i}+\frac{1}{\lambda_{i}}\right)}=\sum_{k}\left(N_{i k} r_{1}^{p_{i}} e^{-\alpha_{i k} r_{k}}+M_{i k} r_{2}^{p_{i}} e^{-\left(\alpha_{i k}+\frac{1}{\lambda_{i}}\right) r_{2}}\right)
$$

where $N_{i k}$ and $M_{i k}$ are the number of NN and NNN of species $k$ of atom $i$ in the actual alloy. The chemical environment surrounding atom $i$ is reflected in the parameters $\alpha_{i k}$, given by

$\alpha_{i k}=\alpha_{i}+\Delta_{k i}$

where the BFS parameters $\Delta$ (a perturbation on the single-element ECT parameter $\alpha_{i}$ ) describe the changes of the wave function in the overlap region between atoms $i$ and $k$. Once Eq. 10 is solved for the equivalent chemical lattice parameter $a_{i}{ }^{C}$, the BFS chemical energy is then

$\varepsilon_{i}^{C}=\gamma_{i} E_{C}^{i}\left(1-\left(1+a_{i}^{C^{*}}\right) e^{-a_{i}^{c^{*}}}\right)$

where $\gamma_{\mathrm{i}}=1$ if $a_{i}^{C^{*}}>0$ and $\gamma_{i}=-1$ if $a_{i}^{C^{*}}<0$ and where the scaled chemical lattice parameter is given by 
$a_{i}^{C^{*}}=q \frac{\left(a_{i}^{C}-a_{e}^{i}\right)}{l_{i}}$

It is worth noting that the BFS parameter $\Delta_{k i}$ is, in a sense, as much a single-element parameter that describes element $i$ as any of the parameters previously introduced. It describes changes in the electron density in the vicinity of atom $i$ due to the presence of atom $k$. It is, figuratively, a conditioned response of atom $i$ to the presence of atom $k$ and it does not depend on the relative location of these two atoms, as would be the case in the context of a traditional potential approach, where there would be a distance-dependent interaction. The definition of the chemical defect, represented by the r.h.s of Eq. 10, requires all atoms to be located at equilibrium nearest-neighbor distance $a_{i}$.

Finally, as mentioned above, the BFS chemical and strain energy contributions are linked by a coupling function $g_{i}$ which describes the influence of the geometrical distribution of the surrounding atoms in relation to the chemical effects and is given by

$g_{i}=\exp \left(-a_{i}^{S^{*}}\right)$

where the scaled lattice parameter $a_{i}^{S *}$ is defined in Eq. 7.

In this work we used the BFS perturbative parameters $\Delta$ determined following the procedure

outlined in Ref. 9. The pure element parameters $a_{e}, E_{c}, l, \alpha, \lambda$ and the BFS parameters $\Delta_{P d C u}$ and $\Delta_{C u P d}$ used in this study are listed in Table 1.

\section{DESCRIPTION OF SINGLE ELEMENT PROPERTIES}

The accuracy of the BFS method relies heavily on the few parameters that describe each single element (including the perturbative parameters $\Delta$ necessary in the description of the alloys). With the objective of validating these parameters, we devote this section to review the performance of the method in a number of applications regarding the surface structure of single element crystals of $\mathrm{Cu}$ and Pd. The rest of the paper is devoted to examine the behavior of the BFS parameters $\Delta_{C u P d}$ and $\Delta_{P d C u}$ through several applications to Cu-Pd alloys.

\subsection{SURFACE ENERGIES AND SINGLE CRYSTAL SURFACE RELAXATION}


The energy of a free surface plays an important role in several physical and chemical processes such as fracture, catalysis, etc. Experimental measurements of the surface energy are usually at or above room temperature and are subject to errors due to surface-active contaminants and thus have a degree of uncertainty. Lately, there has been an increasing effort on first-principles calculations [15-21] as well as in the area of quantum approximate methods. Both EAM [6] and ECT have been applied to this and other surface properties, with the latter method providing excellent agreement with available experimental data $[5,14]$.

Table 2 lists, for the low roughness faces, the different contributions to the surface energy as defined by ECT. These results are compared with several experimental estimates [28,29] as well as the surface energies obtained by other approaches and first-principles calculations [17,22-27]. The 'rigid' results contain only one contribution, $\sigma_{1}$. This 'volume' term decreases substantially once the top layers are allowed to relax, but some of the decrease in energy is balanced by the finite contributions of the anisotropic terms $\sigma_{2}, \sigma_{3}$ and $\sigma_{4}$. Of these three terms, the largest contribution is provided by the bond-length anisotropy term represented by $\sigma_{2}$, which arises from the contraction of the first interlayer spacing. In all cases, the many-body terms due to bond-angle and face-diagonal anisotropies are very small compared to the leading terms and have no effect on the overall behavior of the relaxation patterns as well as the resulting surface energies $[5,30]$.

Multilayer relaxation means that planes in the surface region display shifts perpendicular to the surface, directed both inward and outward, with or without distortion of the two-dimensional mesh cell. In most cases the relaxation pattern is of an oscillatory character, with the alternating compressions and expansions following trends that depend on the stacking (ABAB..., $\mathrm{ABCABC}$,..., etc.) and whose magnitude decreases in approaching the bulk. The results shown in this section indicate that a quantitative comparison with experiment is often difficult, in that there is a substantial range of results for a given material and surface, even regarding the sign of the change. Similarly, quantitative comparisons with first-principles calculations suffer from a sparsity of results. Therefore, the bulk of our assertions about success in predicting surface relaxation is based mostly on agreement with the magnitude and sign of the changes. Several experimental and theoretical studies have concentrated on the $\mathrm{Cu}$ and $\mathrm{Pd}$ low index surface relaxations [6,23,31-56]. Table 3 summarizes the experimental [31,41,50-52] and theoretical $[6,22,23,40,41,44,45]$ results for low-index faces of $\mathrm{Cu}$ and Pd. The agreement between the ECT 
predictions and other theoretical and experimental results $[31,35-39,42,43,50-52]$ is consistent for all faces of $\mathrm{Cu}$ and $\mathrm{Pd}$.

It is important to address the issue of uncertainties in the prediction of multilayer relaxation. All quantum approximate methods used for simulations of surface structure (ECT, EAM, etc.) $[3,4,22]$ rely either on input data (generally experimentally determined) or on certain approximations for some of the variables of relevance. First principles methods have been used to provide input data when experimental results are inaccurate or unavailable. Either way, the uncertainties in the experimental data used or the choices made in the implementation of first-principles calculations should translate into some degree of uncertainty in the results obtained with the chosen quantum approximate method. In order to estimate the impact of such experimental or theoretical uncertainties in the parameterization of the quantum approximate methods, we will attach an estimate of the possible errors due to any of the reasons mentioned above. Although there is no certain way to determine such errors (after all, the predictions are, within their own framework, exact), we will see that even small changes, of the order of $1 \%$, in the surface energy can generate quite interesting variations in the relaxation schemes predicted. In particular, within the framework of ECT (I [5,14] or II [4]), such small changes in the surface energy can be easily obtained by changing any of the input parameters (lattice constant, cohesive energy, bulk modulus) by comparable amounts, well below the usual experimental errors in the determination of such quantities.

We thus define 'error bars' in such a way that all the intermediate values so obtained predict variations in surface energies within a certain tolerance. As an example, we set the tolerance at $1 \%$ of the equilibrium surface energy, $\sigma_{\mathrm{e}}$. This defines a 'surface' $\sigma\left(\Delta \mathrm{d}_{12}, \Delta \mathrm{d}_{23}\right)$ and the allowed values for these parameters are such that $0.99 \sigma_{\mathrm{e}}<\sigma\left(\Delta \mathrm{d}_{12}, \Delta \mathrm{d}_{23}\right)<1.01 \sigma_{\mathrm{e}}$, where $\Delta \mathrm{d}_{\mathrm{nm}}$ denotes the change in separation between planes $\mathrm{n}$ and $\mathrm{m}$. We take, as an example, the case of $\mathrm{Cu}$ for which there is a consistent agreement between experiment, ECT and other theoretical approaches. In Table 4, we compare results for the multilayer relaxations of the first two interlayer spacings for those cases for which data are available $[33,38,42]$. The inclusion of the theoretical 'error bar', as mentioned above, allows for a better comparison with experiment as it shows that for most cases, small changes in the input parameters of the method may account for the whole range of possible experimental results. 
For completeness, and due to the considerable experimental interest in stepped and kinked surfaces, we include predictions on multilayer relaxations for $\mathrm{Cu}(210)$ and $\mathrm{Cu}(311)$, including both perpendicular and parallel relaxations. as percentages of the corresponding bulk spacings in Table 5, including recent experimental results [57].

\section{BFS ANALYSIS OF THE Cu-Pd SYSTEM}

Having established the validity of the parameterization of $\mathrm{Cu}$ and $\mathrm{Pd}$ by means of a detailed analysis of the surface properties of pure crystals, we now proceed to study the $\mathrm{Cu}-\mathrm{Pd}$ system using BFS. It should be noted that every single application of BFS to the interaction of $\mathrm{Cu}$ and $\mathrm{Pd}$ atoms relies on just one pair of parameters, listed in Table 1 , and that no correction or adjustment is made when dealing with different types of applications.

\subsection{HEAT OF SEGREGATION OF SINGLE SUBSTITUTIONAL ADDITIONS}

Surface and interfacial segregation are phenomena with great technological importance $[58,59]$ as they affect both the chemistry of surfaces and the strength of interfaces. In addition to the considerable experimental effort devoted to segregation [60-71], theoretical studies covering a wide range of techniques $[6,22,72-76]$ have been applied to seek further understanding on the driving mechanisms for segregation. A thorough review of experimental and theoretical results for the surface composition and structure for a large number of binary alloys can be found in Ref. 77 .

The heat of segregation is defined as the difference between the heat of formation of a semiinfinite A crystal with an impurity of atomic species B located at a lattice site on plane $p$ ( $p=0$ is the surface plane) parallel to the surface and the same structure, but with the atom $B$ located in a lattice site in the bulk $(p=b)$. For the derivation of an analytical result, we ignore relaxation effects around the impurity as well as surface relaxation of the host crystal, as detailed in the previous section. This is clearly unrealistic, but it is justified in that it allows for the derivation of simple expressions describing the fundamental process. We consider a cell containing the impurity atom such that the host atoms on the boundary of this cell are 'insensitive' to the presence of the impurity (i.e., the distance is large enough to exceed the range of interactions included in the BFS calculation of the energy). The cell is a sufficiently large piece of the crystal which includes 
the impurity atom in layer $\mathrm{p}$, its surrounding neighbors, and several other atoms located in intermediate layers $q\left(q=0,1, \ldots, p, \ldots, p_{b}\right)$. The heat of segregation can then be understood as the exchange of an impurity atom in layer $p$ with a host atom in the bulk. If this exchange lowers the energy, then the impurity does not segregate to the surface.

If $\Delta E_{[p, X]}$ denote the energy of formation of the computational cell when the atom $X$ is located in layer $p$, then the heat of segregation is

$$
\Delta E_{S}^{(p)}=\Delta E_{[p, B]}-\Delta E_{[p, A]}
$$

After some algebra, an expression which depends only on individual strain and chemical energy contributions can be obtained,

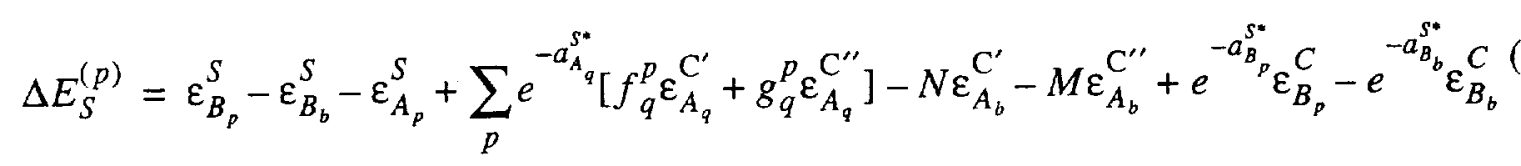

where $\varepsilon_{X_{p}}^{S}\left(\varepsilon_{X_{p}}^{c}\right)$ is the strain (chemical) energy of an atom $\mathrm{X}$ in layer $\mathrm{p}, f_{q}^{p}\left(g_{q}^{p}\right)$ denotes the number of $\mathrm{NN}(\mathrm{NNN})$ than an atom in layer $p$ has in layer $q$ and where $\varepsilon_{X_{p}}^{\mathrm{C}^{\prime}}\left(\varepsilon_{X_{p}}^{\mathrm{C}^{\prime \prime}}\right)$ is the chemical energy of an atom $X$ in layer $p$ that is a NN (NNN) of the impurity atom B. The scaled lattice constant $a^{S^{*}}$ is given by

$$
a_{X_{p}}^{S^{*}}=\frac{\left(a_{X_{p}}^{S}-a_{e}^{X}\right)}{l_{X}}
$$

Including relaxation would render this expression unmanageable, as it would include every atom in the cell as well as a substantial number of variables (interplanar spacings for the surface planes and interatomic relaxations around the impurity in the bulk). It is interesting to examine this simplified result as it provides a simple, analytical way to isolate the different mechanisms involved in segregation and their influence in the heat of segregation. Eq. 16 shows that two distinct contributions are responsible for segregation: a strain term,

$$
\Delta S_{S}^{(p)}=\varepsilon_{B_{p}}^{S}-\varepsilon_{B_{b}}^{S}-\varepsilon_{A_{p}}^{S}
$$


and a chemical term

$$
\Delta C_{s}^{(p)}=\Delta E_{S}^{(p)}-\Delta S_{S}^{(p)}
$$

both defined in the context of BFS. One immediate conclusion regarding the driving mechanism is drawn from the predictions from these last two equations, which are summarized for a large number of host-impurity systems in Table 6: the strain energy contribution consistently has the same sign as the whole quantity, indicating that the segregation trends obtained from Eq. 16 are dictated by the relative value of the strain energy, as one would expect. Moreover, two different contributions can be selected from the different terms that make up the strain energy: $\varepsilon_{B_{p}}^{S}-\varepsilon_{B_{b}}^{s}$, directly related to the structural strain induced by the presence of the impurity, and $\varepsilon_{B_{p}}^{S}-\varepsilon_{A_{p}}^{S}$, which indirectly reflects the difference in surface energies of the two participating species (note that, as defined, these are not competing mechanisms). The first quantity does not follow the correct trend for the total BFS energy in all cases, although it tends to do so as the lattice mismatch between species A and B increases. We then conclude that only when the lattice mismatch is sufficiently large so as to make $\Delta S_{S}^{(0)}<0$ this effect can be taken as the one responsible for identifying the segregating species. The 'surface energy' term, $\varepsilon_{B_{p}}^{S}-\varepsilon_{A_{p}}^{S}$, consistently reproduces the same trends as $\Delta E_{S}$ as a whole, as well as experimental trends. These results are displayed in Table 6: the first column shows typical experimental predictions [76] for the sign of $\Delta E_{S}^{(0)}$. The second column displays the corresponding results obtained from Eq. 16 and the last three columns show the sign of $\Delta S_{S}^{(0)}, \varepsilon_{B_{0}}^{S}-\varepsilon_{B_{b}}^{S}$ and $\varepsilon_{B_{0}}^{S}-\varepsilon_{A_{0}}^{S}$. A negative sign in the first two columns indicates that the impurity atom segregates to the surface. It should be noted that as we move down to planes below the surface, one would expect chemical effects to dominate as the influence of the surface is lessened, resulting into a more delicate balance between $\Delta \mathrm{S}$ and $\Delta \mathrm{C}$, which could translate into segregation patterns where certain layers are enriched and other are not, sometimes in an oscillatory fashion.

Unlike most other systems for which theoretical or experimental evidence is available, $\mathrm{Cu}-\mathrm{Pd}$ is inherently uncertain: theory and experiment are equally divided with respect to the choice of the segregating species. A recent theoretical study even finds a segregation reversal where for $\mathrm{Cu}_{\mathrm{x}} \mathrm{Pd}_{1-\mathrm{x}}$ disordered alloys, the segregating species varies with composition [60]. However, the overall agreement between BFS and experiments for other systems suggests that further experi- 
mental work might confirm the theoretical predictions. It should be noted, however, that small variations in the input parameters $\Delta_{A B}$ and $\Delta_{B A}$ are sufficient, in some cases when the heat of segregation is small, to reverse the predicted behavior. For the particular case of $\mathrm{Cu}-\mathrm{Pd}$ studied in this work, the BFS parameters were obtained by fitting to the experimental heat of solution in the dilute limit, which is directly related to the physical phenomenon represented by the heat of segregation. We then suggest that when the heat of segregation predicted by BFS is small, uncertainties in the experimental input used (and thus $\Delta_{A B}$ and $\Delta_{B A}$ ) must be questioned, before those predictions are accepted.

\subsection{SURFACE ALLOYS}

The emerging field of surface alloys, i.e., alloys that form only on a surface typically including from one to a few layers, provides a wealth of new and unexpected phenomena where atomistic modeling can be of help in understanding the experimental observations [78-83]. The level of detail needed to properly describe the observed features, or to predict the behavior of unknown systems, imposes a severe requirement not only on the strength of the theory, but also on the parameters used. Beyond the ability of any given method to properly describe surfaces with the same accuracy with which it describes bulk features, it is also necessary that the method has enough resolution to be able to successfully distinguish the complex patterns observed in surface alloys, which clearly includes surface phenomena as well as the interactions between two or more different elements. In this section, we focus on the deposition of $\mathrm{Pd}$ on $\mathrm{Cu}(100)$ and $\mathrm{Cu}(110)$, and $\mathrm{Cu}$ on $\mathrm{Pd}(110)$, highlighting the fact that in spite of the nature of the problem, no changes or adjustments are made on the parameters used. These three surface alloys, for which there is abundant experimental work [84-90], display substantially different patterns: (a) $\mathrm{Pd} / \mathrm{Cu}(100$ ) is characterized by a c(2x2) structure for low Pd coverage [84-87]; (b) $\mathrm{Pd} / \mathrm{Cu}(110)$ shows the formation of $\mathrm{Pd}-\mathrm{Cu}$ chains, resulting into a $\mathrm{Cu}_{3} \mathrm{Pd}$ one-layer surface alloy [88] and (c) $\mathrm{Cu} / \mathrm{Pd}(110)$ features monoatomic linear chains oriented in the [110] direction for low $\mathrm{Cu}$ coverage $[89,90]$.

The results shown in this section were obtained from implementing a calculational procedure based on the determination of 'catalogues' of atomic configurations describing a large portion of the substrate and the adlayer [91,92]. The energy of each configuration is computed with the BFS method and the surface alloy formation process is then reconstructed by linking the states with 
lower energy, as the coverage increases. As information on higher energy states is also available, an advantage of this procedure lies in the insight that it provides on metastable states and therefore on alternate ordering patterns. However, being that there is no specific set of rules for constructing such catalogues, the effectiveness of this approach is directly related to our ability to include in these catalogues all the relevant configurations.

A catalogue of configurations is built focusing on the symmetries of the substrate, but general enough to include most of the possible growth patterns. For the sake of simplicity, these configurations do not include individual or collective atomic relaxations, and all the calculations are performed at zero temperature. Both conditions can be relaxed but, given the objective of this paper (i.e., the transferability of the BFS parameters for $\mathrm{Cu}$ and $\mathrm{Pd}$ to any bulk or surface problem), a full treatment of relaxations and thermal effects would distract from the main goal.

(a) $\mathrm{Pd} / \mathrm{Cu}(100)$ : A catalogue of configurations for Pd coverage up to $0.5 \mathrm{ML}$ was built, including states with $\mathrm{Pd}$ atoms in surface sites substituting for surface $\mathrm{Cu}$ atoms, as well as sites in the overlayer [91]. Starting with low Pd coverage, it is seen that Pd atoms insert themselves in the (100) surface layer in nearest-neighbor sites along the [010] and [100] directions, leading to a $c(2 \times 2)$ structure at higher coverages. The ejected $\mathrm{Cu}$ atoms either migrate to nearby steps or nucleate on top of alloyed areas resulting in subsurface $\mathrm{Pd}$. The preference for the formation of a $\operatorname{Pd} c(2 \times 2)$ structure is highlighted by the fact that alternative ordering patterns (even those that show a slight departure from the $c(2 \times 2)$ ordering) are much higher in energy and therefore unlikely to occur. However, the low-lying energy states (all including $c(2 \times 2)$-like patterns) also include states where some $\mathrm{Pd}$ atoms interdiffuse below the surface layer, a trend enhanced for higher Pd coverage. While this feature hints to the possibility of much more complex alloying patterns for Pd coverage close to $0.5 \mathrm{ML}$, it also establishes a limit for the validity of the restrictions imposed in the current calculation, as subsurface $\mathrm{Pd}$ penetration would require a full treatment of collective atomic relaxations. In fact, experiment indicates a clock-rotated phase with the $c(2 \times 2)$ $p 4 g$ symmetry [84], consistent with a substantial displacement of the subsurface atoms [84]. In spite of the fact that these are static calculations, the motion of $\mathrm{Cu}$ islands or $\mathrm{Cu}$ atoms in the adlayer can be modeled by analyzing configurations where $\mathrm{Cu}$ resides on top of surface Pd atoms, attached to a nearby step, or somewhere in between. The results show that $\mathrm{Cu}$ migrates to nearby steps, with decreased mobility for increasing Pd coverage (i.e., the difference in energy between 
alloyed spots and steps decreases). Summarizing the results for $\mathrm{Pd} / \mathrm{Cu}(100)$, the BFS calculations successfully reproduce all the features observed experimentally up to 0.5 ML Pd coverage $[84,85]$ : the alloying of Pd atoms in the surface in the [010] and [100] directions, the nucleation of $\mathrm{Cu}$ islands on top of alloyed areas, the decreased mobility of $\mathrm{Cu}$ islands with increasing Pd coverage, the formation of a $\mathrm{c}(2 \times 2)$ phase as the chains converge, and the interplay between the $\mathrm{c}(2 \times 2)$ phase and the initiation of second layer growth at increasing coverages before the $c(2 \times 2)$ is fully completed. Fig. 2 illustrates this last feature, by studying the evolution of the energy of formation of selected states, namely, the $\mathrm{c}(2 \times 2)$ ground state and the configurations corresponding to the $\mathrm{Pd}$ atom in the near-surface layers.

(b) $\mathrm{Pd} / \mathrm{Cu}(110)$ : Once again, it is seen that $\mathrm{Pd}$ atoms exchange sites with $\mathrm{Cu}$ surface atoms [88-92]. However, the particular features of an fcc (110) surface turn this Pd surface atom into a strong nucleation point for $\mathrm{Cu}$ atoms in the overlayer, which in turn stimulates the formation of $\mathrm{Cu}$ chains in the overlayer, as opposed to $\mathrm{Cu}$ islands attached to alloyed surface regions. An energy level diagram representing this process is shown in Fig. 3. Configurations with neighboring Pd atoms in surface sites are substantially higher in energy than those where Pd atoms interact only through $\mathrm{Cu}$ adatoms. In agreement with experiment, no additional features, like Pd penetration in subsurface layers, are observed. For increasing Pd coverages the growth pattern remains the same, resulting in the formation of $\mathrm{Cu}$ chains in the adlayer, coupled with alternating $\mathrm{Cu}$-Pd$\mathrm{Cu}$ surface atoms. Not surprisingly, this experimentally observed pattern [88] corresponds to the [010] direction in a (110) termination of an $\mathrm{Ll}_{2} \mathrm{Cu}_{3} \mathrm{Pd}$ alloy. The lack of alternate ordering patterns that compete energetically with the one observed, leads to the conclusion that for higher $\mathrm{Pd}$

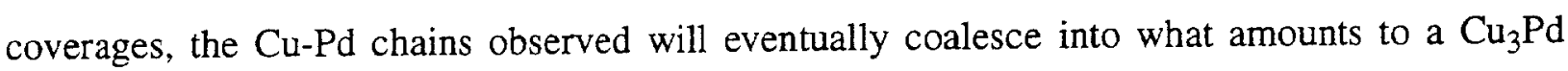
(110) surface, with full coverage of $\mathrm{Cu}$ with a 2:1 $\mathrm{Cu}$ :Pd ratio, as observed experimentally.

(c) $C u / P d(110)$ : Experimental results for this system clearly show that the observed growth pattern is substantially different from the systems where Pd is deposited on a Cu substrate [89,90]. The energy level diagram shown in Fig. 4 indicates that $\mathrm{Cu}$ does not substitute for Pd surface atoms. Instead, the state of minimum energy corresponds to the $\mathrm{Cu}$ atom attached to a Pd step edge in the [110] direction, with the step edge acting as a nucleation center favoring the growth of 1D Cu chains. While this trend is also observed at higher coverages, the preference for separate anchoring points in the step edge is diminished at higher coverages, suggesting a $2 \mathrm{D}$ growth of the linear chains. Still, it is expected that a clear separation between $1 \mathrm{D}$ chains will dominate the low 
coverage regime. The energy levels in Fig. 4 correspond to selected configurations with increasing $\mathrm{Cu}$ coverage, clearly showing that $\mathrm{Cu}$ atoms are always trapped by a substrate edge or existing $\mathrm{Cu}$ islands. The $\mathrm{Cu}$ 'fingers' in the [1]0] direction are observed experimentally for room temperature and low $(<0.1 \mathrm{ML}) \mathrm{Cu}$ coverage, as well as the formation of $2 \mathrm{D}$ islands for higher Cu coverages, consistent with the modeling results.

\subsection{CuPd SOLID SOLUTION}

As shown in Fig. 1, a continuous solid solution is found at high temperatures. Measurements of the lattice spacings of the solid solution date back to 1932 [93]. Lattice spacings were said to be accurate to $\sim 0.05 \%$. In order to obtain BFS predictions for the iattice parameter of the solid solution, it is convenient to use a simple algorithm based on the cluster expansion method [94], which allows for the calculation of finite temperature lattice parameter values from information describing certain zero-temperature ordered structures (regardless of the fact if these structures are found in nature or not). In this approach, the equilibrium properties of $\mathrm{Cu}_{3} \mathrm{Pd}, \mathrm{CuPd}$ and $\mathrm{CuPd}_{3}$ in the $\mathrm{Ll}_{2}, \mathrm{Ll}_{0}$ and $\mathrm{Ll}_{2}$ structures, respectively, are computed with the BFS method as a function of volume. Following the Connolly-Williams scheme [95], these results yield information of the different many-body interactions that characterize the system. Once these potentials are known, it is straightforward to obtain the energy vs. volume curve for the disordered solid solution.

We start by computing the formation energy of the five basis clusters needed, within the tetrahedron approximation (only clusters consisting of $N N$ ). These simple clusters are the building blocks of the corresponding ordered fcc alloys $A_{m} B_{4-m}$. The formation energy is then

$$
\Delta E_{m}=E_{m}(r)+\frac{m}{4} E_{4}\left(a_{e}^{A}\right)-\left(1-\frac{m}{4}\right) E_{0}\left(a_{e}^{B}\right)
$$

where $a_{e}^{A}$ and $a_{e}^{B}$ are the equilibrium lattice constants of pure A and B metals, $E_{4}\left(a_{e}^{A}\right)=E_{C}^{A}$, and $E_{0}\left(a_{e}^{B}\right)=E_{C}^{B}$. With these definitions, $E_{m}(r)$ represents the binding energy curve of the ordered alloy as function lattice spacing. The energy of formation of the disordered structures $A_{x} B_{1-x}$ as a function of the ordered ones [95] can be computed with 


$$
\left.\Delta E_{D} r, x\right)=\sum_{m=0}^{4}\left(\begin{array}{l}
4 \\
m
\end{array}\right) x^{m}(1-x)^{4-m} \Delta E_{m}(r)
$$

The lattice parameter for the disordered alloy $A_{x} B_{1-x}$ is then given by the value of $\mathrm{x}$ which minimizes $\Delta E_{D}$, and the energy of formation is the corresponding minimum value of $\Delta E_{D}$. The values for the lattice parameter as a function of composition are shown in Table 7. Table 8 shows the energy of formation as a function of concentration.

\subsection{ORDERED STRUCTURES}

The weaith of experimental studies of surface relaxation on pure metallic surfaces is not matched for alloys [97]. However, in spite of the small number of experimental studies [98-102], there seems to be a slow but sure progress in the field, as the available theoretical tools for modeling become more accurate.

The Cu-Pd phase diagram shown in Fig. 1 exhibits an $\mathrm{LI}_{2}$ ordered phase at low temperatures. In this section, we apply BFS to the calculation of the surface structure of $\mathrm{Cu}_{3} \mathrm{Pd}$.

\subsubsection{Multilayer relaxation and surface structure of $\mathrm{Cu}_{3} P d$}

The lattice parameter of the $\mathrm{Cu}_{3} \mathrm{Pd}$ phase, as a function of composition, can be computed from the BFS predictions for $\Delta E_{m}(r)$, for $\mathrm{m}=3$ (see Eq. 20 ). The calculation of the energy vs. volume curve is a straightforward calculation, as all $\mathrm{Cu}$ atoms are equivalent:

$$
\Delta E_{m}(r)=\frac{1}{4}\left(3 e_{C u}+e_{P d}\right)
$$

where $e_{C u}$ and $e_{P d}$ represent the contributions of $\mathrm{Cu}$ and $\mathrm{Pd}$ atoms, respectively, computed using the formalism introduced in Sec. 2. By minimizing $\Delta \mathrm{E}$ with respect to the lattice parameter, the equilibrium spacing of the $\mathrm{L}_{2}$ phase is thus obtained. 
The $\mathrm{Cu}_{3} \mathrm{Pd}$ phase is simple enough to allow for a straightforward application of BFS for the determination of surface energies and multilayer relaxation patterns. Previous studies using BFS of $\mathrm{A}_{3} \mathrm{~B}$ structures $\left(\mathrm{Ni}_{3} \mathrm{Al}, \mathrm{Cu}_{3} \mathrm{Au}\right)$ [103] have shown that the methodology provides reliable results for the calculation of surface structure in comparison with experiment, in particular, the determination of the individual displacement of surface atoms of different sizes in mixed-composition surfaces.

There are two possible terminations for the $(100) \mathrm{Ll}_{2}$ structure of a certain $\mathrm{A}_{3} \mathrm{~B}$ alloy: a mixed composition $(1: 1 \mathrm{~A}: \mathrm{B})$ plane alternating with a pure $\mathrm{A}(1: 0 \mathrm{~A}: \mathrm{B})$ plane, giving an overall stoichiometry ( $3: 1 \mathrm{~A}: \mathrm{B})$, and a pure A plane alternating with mixed-composition planes. These two possible bulk truncations are also possible for the (110) surface, whereas the (111) truncation is always stoichiometric $(3: 1 \mathrm{~A}: \mathrm{B})$. We will assume that the lattice parameter $\mathrm{a}$ is the one previousiy determined for a bulk ordered alloy $\mathrm{A}_{3} \mathrm{~B}$.

While no experimental data for $\mathrm{Cu}_{3} \mathrm{Pd}$ exist, we provide a foundation for the validity of our predictions by discussing results for $\mathrm{Ni}_{3} \mathrm{Al}$ and $\mathrm{Cu}_{3} \mathrm{Au}$, which have a similar structure, in comparison with other experimental or theoretical results.

For $\mathrm{Cu}_{3} \mathrm{Au}$, relaxed surface energies are shown as obtained with Finnis-Sinclair many-body potentials [104] and BFS. Both methods predict, as expected, lower surface energies for the mixed composition (100) and (110) truncations. This feature has been experimentally proven via a lowenergy ion scattering study which detected equal parts of $\mathrm{Cu}$ and $\mathrm{Au}$ in the top layer [105]. BFS and FS results also agree on the relative change in surface energy once the topmost layers are allowed to relax, in spite of the fact that the FS values are 50\% smaller than the BFS ones. As is also to be expected, the surface energies of (100) 1:0 and (110) 1:0 faces are comparable to the corresponding values for single $\mathrm{Cu}$ crystals. In what follows, the relaxations are indicated as the percentage change in interlayer spacing from the unrelaxed case to the one measured from the relaxed position to the unrelaxed location of the plane immediately below. For the $\mathrm{Cu}_{3} \mathrm{Au}(100)$ 1:1 Cu:Au case, the results imply a rippling of $0.148+/-0.025 \AA$, which amounts to $3.97 \%$ of the lattice parameter determined for this alloy $(3.73 \AA)$. This result compares very well with the $3.77 \%$ rippling obtained using FS potentials [104]. A similar situation is found for the (110) 1:1 $\mathrm{Cu}: \mathrm{Au}$ surface, where we find the rippling to be $4.2 \%$ of the lattice parameter, whereas FS potentials predict a rather small change of $1.9 \%$. For the mixed composition (111) 3:1 Cu:Au surface, BFS predicts a rippling of $4.6 \%$, thus agreeing with FS results and experimental evidence that the 
$\mathrm{Au}$ atoms are farther out than the neighboring $\mathrm{Cu}$ atoms in mixed-composition surfaces. For $\mathrm{Ni}_{3} \mathrm{Al}$, the following values for the gap between $\mathrm{Ni}$ and $\mathrm{Al}$ atoms in the mixed composition (100), (110) and (111) surfaces is found: $0.12 \AA, 0.09 \AA$ and $0.16 \AA$, respectively. A similar trend, but with somewhat smaller values for the rippling are obtained from EAM [106]: $0.09 \AA, 0.06 \AA$ and $0.07 \AA$, respectively. A different EAM calculation [107] predicts a $0.06 \AA$ separation between $\mathrm{Ni}$ and $\mathrm{Al}$ atoms in all three surfaces. Recent LEED data [99] predict a distance of $0.02 \AA$ between $\mathrm{Ni}$ and $\mathrm{Al}$ atoms for the (100) surface. Based on the ability of BFS to describe the surface structure of the $\mathrm{Ll}_{2} \mathrm{Ni}_{3} \mathrm{Al}$ and $\mathrm{Cu}_{3} \mathrm{Au}$, Table 9 lists similar predictions for $\mathrm{Cu}_{3} \mathrm{Pd}$.

\subsubsection{Order-disorder transition: $\mathrm{Cu}_{3} \mathrm{Pd}$}

For the study of order-disorder transitions, we make use of Monte Carlo - Metropolis large scale simulations. Following the traditional algorithm, an initial, random, structure is generated by assigning $\mathrm{Ni}, \mathrm{Al}$ and $\mathrm{Fe}$ atoms to rigid (i.e., no individual relaxations) lattice sites in a 1024-atom computational cell with periodic boundary conditions in all three directions. The kinetics of ordering with decreasing temperature are represented by the exchange of randomly chosen pairs of atoms of different atomic species throughout the cell. The difference in energy of formation of the cell before and after the exchange, $\Delta \mathrm{E}$, is used to determine the likelihood that this exchange can take place. If $\Delta \mathrm{E}<0$, the exchange is readily accepted. If not, it is assigned a probability $\exp (\Delta \mathrm{E} / \mathrm{kT})$, where $\mathrm{k}$ is Boltzman's constant and $\mathrm{T}$ is the temperature. For a fixed value of $\mathrm{T}$, the cell is allowed to evolve until its total energy stabilizes. The process is repeated for decreasing values of $T$, until a final, equilibrium, state is reached (i.e., every possible distribution of atoms is considered until the one with the lowest possible energy is found). The algorithm favors the ideal atomic distribution consistent with an infinite annealing time. We call this procedure all-swaps (AS) [108], as any atomic exchange is eventually considered and the actual ground state of the system is reached.

For the purpose of this work, where simulations are only meant to illustrate and expand on the results obtained from the spectral analysis, it is convenient to introduce an alternative approach to aid in the understanding and interpretation of some features that characterize the results of the standard Monte Carlo - Metropolis procedure. While traditionally used for the determination of ground state properties, the AS approach does not necessarily provide the best means for compar- 
ison with experiment, due to its unrealistic treatment of atomic interdiffusion. Moreover, AS does not constitute a proper description of temperature effects as $\mathrm{T}$ enters in the simulations only through the probability factor $\mathrm{kT}$. This results in an incorrect scaling between temperature and the observed ordering. The unlimited range of the atomic exchanges allows for ordering at very high temperatures, thus proving highly inefficient for a proper analysis of order-disorder transitions.

These limitations can be avoided, within the framework of an approximate variation of the standard Monte Carlo - Metropolis algorithm, solely designed to focus on the modeling of the low temperature behavior of individual atoms. A second simulation approach is therefore introduced, particularly designed to avoid these limitations [109]. In this algorithm, which we denote NN, we limit the range of atomic exchanges to pairs of atoms located at nearest-neighbor distances. Moreover, we modify the probability criterion by defining it in terms of the ratio between the energy necessary for the exchange, $\Delta E$, and the available thermal energy, given by

$$
E_{T h}=9 N k T\left(\frac{T}{\Theta_{D}}\right)^{3} \int_{0}^{\frac{\Theta_{D}}{T}} \frac{x^{3}}{e^{x}-1} d x
$$

where $\Theta_{D}$ is the Debye temperature [110]. In this approach, the actual ground state of the system is not necessarily reached, as different atoms could eventually get 'trapped' at low temperatures, thus unable to reach their true equilibrium positions. Together, AS and NN simulations supplement each other, providing insight not only on the nature of the thermodynamic ground state (AS) but also on the most likely features observed experimentally (NN). We stress, however, that the $\mathrm{NN}$ approach does not rely on a rigorous statistical mechanics foundation as AS does. It is, at best, a simple but approximate way to model the evolution of the system and therefore illustrate the consequences of the basic features of the energy spectrum that is obtained from the analytical calculations (i.e., small energy gaps between the low-lying energy levels).

The results of simulations using the $\mathrm{NN}$ algorithm and the same parameters for fcc $\mathrm{Cu}$ and $\mathrm{Pd}$ used throughout this paper are shown in Figs. 5-8. Four alloys were studied: $\mathrm{Cu}_{70} \mathrm{Pd}_{30}$ (Fig. 5), $\mathrm{Cu}_{75} \mathrm{Pd}_{25}$ (Fig. 6), $\mathrm{Cu}_{82} \mathrm{Pd}_{18}$ (Fig. 7) and $\mathrm{Cu}_{88} \mathrm{Pd}_{12}$ (Fig. 8). Starting from a (high-temperature) random distribution, all 1008-atom cells were given the same temperature treatment, namely, descending $100 \mathrm{~K}$ steps to room temperature. A few selected views of the computational cell are shown in Figs. 5-8, highlighting a) disorder at high temperature, b) an ordering trend usually in 
the range 700-900 $\mathrm{K}$ and $\mathrm{c}$ ) the formation of specific ordered structures for different compositions. For $\mathrm{Cu}_{70} \mathrm{Pd}_{30}$, hints of order are clearly visible at $800 \mathrm{~K}$, leading to a fully ordered $\mathrm{L}_{2}$ structure at $400 \mathrm{~K}$. It should be noted that, due to the relatively low influence of NNN interactions in the energy of the cell, it is likely that the computational cell will display numerous 'antiphase boundaries', separating regions of the cell with the same type of ordering, as is the case in Fig. 5.e. The intermediate steps in the cooling of a $\mathrm{Cu}_{75} \mathrm{Pd}_{25}$ alloy are shown in Fig. 6. Once again, there is an order-disorder transition in the $800-900 \mathrm{~K}$ range, higher than that observed for the $\mathrm{Cu} 70 \mathrm{Pd} 30$ alloy. In this case, however, there is a strong ordering trend leading to a nearly perfect $\mathrm{L}_{2} \mathrm{Cu}_{3} \mathrm{Pd}$ alloy at $400 \mathrm{~K}$. The phase field of the $\mathrm{L}_{2}$ structure extends to both sides of the 25 at.\% $\mathrm{Pd}$ alloy. Simulations for a $\mathrm{Cu}_{88} \mathrm{Pd}_{12}$ alloy were made and similar results were found in terms of the transition temperature, in that it remains substantially constant in the $800 \mathrm{~K}$ range. Selected steps in the cooling process of this $\mathrm{Cu}_{82} \mathrm{Pd}_{18}$ alloy are shown in Fig. 7, showing the formation of a perfect $\mathrm{Ll}_{2}$ phase. One last case, $\mathrm{Cu}_{88} \mathrm{Pd}_{12}$, at the edge of the $\mathrm{Ll}_{2}$ phase field (see Fig. 1), is shown in Fig. 8. In excellent agreement with the experimental phase diagram, there is very little evidence of ordering in this case.

\subsubsection{Order-disorder transition: $\mathrm{CuPd}$}

In order to study order-disorder transitions, all possible ordered structures, for different compositions, should be evaluated. In doing so, the ordered structure with the lowest energy can be determined for each composition. To achieve this goal a demanding computational effort is required which, in spite of the simplicity of BFS, greatly exceeds the scope of this paper.

BFS requires that each atom has to be parameterized in the symmetry of the alloy in which it is found. Until now, we have used fcc parameters for $\mathrm{Cu}$ and $\mathrm{Pd}$ as every study described in this paper has dealt with the fcc phase of these elements. If we were to follow the brute force approach mentioned above for the determination of the phase diagram, parameters for $\mathrm{Cu}$ and $\mathrm{Pd}$ should be obtained for each and every possible symmetry. While this is certainly possible, we will restrict our study to just two possible symmetries: fcc and bcc. For the bcc phase of $\mathrm{Cu}$ or $\mathrm{Pd}$, there is no experimental input that can be used to perform the appropriate parameterization, therefore, the needed input parameters have to be obtained from a different source. This problem, similar to the one encountered in previous studies of alloy phases different from those of the pure constituents 
[10] was solved by computing the necessary parameters from first-principles calculations, using the Linear-muffin tin orbital method (LMTO) [111] for bcc elements. Regardless of what firstprinciples method is used, there is no need to use experimental input at all, as all parameters -even those corresponding to the ground state of the element- can be generated by the same method, thus introducing a high level of consistency in terms of the sources for input parameters for the method. However, while first-principles predictions of lattice parameters are generally accurate, that is not the case for the cohesive energies and the bulk modulus.

Being the only alternative to be able to model bcc $\mathrm{Cu}$, $\mathrm{Pd}$ or Cu-Pd alloys, it is then necessary to replace the fcc $\mathrm{Cu}, \mathrm{Pd}$ and the BFS parameters $\left(\Delta_{P d C u}, \Delta_{C u P d}\right)$ used in previous sections (obtained from experimental input) with a new set purely derived from first-principles calculations. Otherwise, there would be no consistency in the comparison of bcc- and fcc-based alloy structures. While this last statement seems to contradict the original claim in this paper, i.e., the universality of the parameters used, it should be noted that nothing prevents from always using, in every application, the parameters derived from first-principles instead of the ones obtained from experimental input, as done in all the previous sections in this paper. In fact, most of the recent work using BFS for the study of high-temperature ordered intermetallic alloys has been done solely with theoretical parameters.

To compute the input parameters for a single element (in any given symmetry), we will use the approach used in Ref. 10, where the energy vs. volume curve is built from first-principles calculations, and then fitted to the UBER [7], from which the cohesive energy, equilibrium lattice parameter and bulk modulus can be extracted. To compute the BFS parameters $\Delta_{A B}$ and $\Delta_{B A}$, it is necessary to build the binding energy curve for a given ordered structure and extract, as in the case of the single element, the cohesive energy per atom, the equilibrium lattice parameter and the bulk modulus. Once these quantities are known, using the resulting energy of formation and equilibrium lattice parameter, the BFS chemical energies can be computed analytically. It is then possible to find the values of $\Delta_{A B}$ and $\Delta_{B A}$ that would result in those values of the BFS chemical energies. This straightforward procedure is further simplified by the fact that in order to build the binding energy curve for the ordered phase, it is not necessary to compute a large number of points. It is actually sufficient to compute just two points on that curve, or as many points as nonequivalent atoms are needed to describe the structure. For $\mathrm{L}_{0}, \mathrm{~L}_{2}$ and $\mathrm{B} 2$ structures, for exam- 
ple, just two points are needed. To fix ideas, consider the B2 structure of CuPd. Being that all $\mathrm{Cu}$ (and Pd) atoms are equivalent, the energy of formation can be simply written as

$$
\Delta H_{B 2}(a)=\frac{1}{2}\left(\varepsilon_{C u}(a)+\varepsilon_{P d}(a)\right)
$$

where $e_{C u}\left(e_{P d}\right)$ is the BFS contribution of a generic $\mathrm{Cu}(\mathrm{Pd})$ atom as a function of the lattice parameter of the ordered phase:

$$
\varepsilon_{A}(a)=\varepsilon_{A}^{S}(a)+g_{A}(a) \varepsilon_{A}^{C}
$$

The determination of the chemical energies (independent of volume) requires the knowledge of two points only in the binding energy curve of the alloy, $E^{\prime}\left(a_{1}\right)$ and $E^{\prime}\left(a_{2}\right)$ :

$$
\Delta H_{i}=\Delta H\left(a_{i}\right)=E^{\prime}\left(a_{i}\right)-\frac{1}{2}\left(E_{C}^{A}+E_{C}^{B}\right)+\frac{1}{2}\left(\varepsilon_{A}^{C}+\varepsilon_{B}^{S}\left(a_{i}\right)+g_{B}\left(a_{i}\right) \varepsilon_{B}^{C}\right)
$$

for $i=1,2$, from which the chemical energies can be obtained. In terms of the alloy energies $E^{\prime}\left(a_{A}\right)$ and $E^{\prime}\left(a_{B}\right)$ and the pure element parameters, the chemical energies are then:

$$
\varepsilon_{A}^{C}=\frac{g_{B}\left(a_{2}\right)\left(2 \Delta H_{1}-S_{1}\right)-g_{B}\left(a_{1}\right)\left(2 \Delta H_{2}-S_{2}\right)}{g_{A}\left(a_{1}\right) g_{B}\left(a_{2}\right)-g_{A}\left(a_{2}\right) g_{B}\left(a_{1}\right)}
$$

and a similar expression for the chemical energy of atom B, where

$$
S_{i}=\left(e_{A}^{S}\left(a_{i}\right)+e_{B}^{S}\left(a_{i}\right)\right)
$$

It is then possible to determine the scaled lattice parameter of the (chemical) equivalent crystal and from there, the values of $\Delta_{C u P d}$ and $\Delta_{P d C u}$ that are consistent with those results. 
Once the chemical energies are known, it is also possible to determine the equilibrium lattice parameter of the ordered phase by minimizing the expression for the energy of formation of the ordered structure, leading to the following transcendental equation for $a_{0}$ :

$$
\sum_{A, B} \frac{g_{i}}{l_{i}}\left(E_{C}^{i} a_{i}^{S^{*}}-\varepsilon_{i}^{\bar{C}}\right)=0
$$

where

$$
a_{i}^{S^{*}}=\frac{q}{l_{i}}\left(a_{0}-a_{i}\right)
$$

The energy of formation of the ordered phase, $\Delta H_{0}$, is then

$$
\Delta H_{0}=\Delta H\left(a_{0}\right)
$$

Table 10 displays the pure element parameters ( $\mathrm{Cu}$ and $\mathrm{Pd}$ in the bcc or fcc phase), as obtained from first-principles calculations and, in the case of the fcc phase, from experiment. Table 11 compares the values of $\Delta H_{O}$ and $a_{O}$ obtained from linearized-augmented plane wave method (LAPW) calculations [112] for the $\mathrm{L}_{0}$ phase with those obtained experimentally. Also, the LAPW parameters for the $\mathrm{B} 2$ phase of CuPd are shown. For completeness, LMTO parameters for both phases are also included in all cases. The resulting values for the BFS interaction parameters are $\Delta_{C u P d}=-0.03248 \AA^{-1}$ and $\Delta_{P d C u}=0.04073 \AA^{-1}$ (for bcc alloys) and $\Delta_{C u P d}=-0.02621 \AA^{-1}$ and $\Delta_{P d C u}=-0.04916 \AA^{-1}$ (for fcc) alloys.

It is clear now that with the availability of bcc and fcc parameters for $\mathrm{Cu}$ and $\mathrm{Pd}$, it is possible to examine the competition between the $\mathrm{B} 2$ and the $\mathrm{L} 1_{0}$ phases of CuPd. Table 11 clearly shows that the B2 structure has a substantially lower energy of formation. It is also possible to study the transition order-disorder by means of Monte Carlo simulations

The analysis of the order-disorder transition for CuPd involves also the fact of a phase change, from an fcc disordered solid solution to a bcc B2 ordered phase. Only in this situation, and for the sake of consistency, we will compare results of NN simulations of bcc and fcc cells using, in both cases, first-principles LAPW parameters. Using experimentally determined parameters for the fcc case, as done for the $\mathrm{L}_{2}$ phase, and theoretically determined bcc parameters would not constitute 
a proper comparison due to the noticeable differences in cohesive energies, which would translate into an inaccurate determination of the ordering process. This can be seen in Fig. 9, which shows the energy of the fcc cell as a function of temperature (once the energy of the cell is stabilized for any given temperature). The curve obtained using LAPW parameters (open squares) predicts a positive energy of formation of the disordered fcc phase, while the one obtained using the experimental parameters (open disks) predict a negative energy of formation for the whole range of temperatures considered, as expected. However, it is also clear that both curves share, overall, the same features (i.e., temperature dependence). The transition temperatures for the $\mathrm{Cu}_{3} \mathrm{Pd}$ orderdisorder transition are correctly predicted by the simulation using experimental parameters. The corresponding transition temperature from the LAPW-based simulation is noticeably lower. A similar situation occurs for $\mathrm{Cu}_{60} \mathrm{Pd}_{40}$ alloys (in the fcc phase): once again both curves display similar features (open disks and squares in Fig. 13), with different predictions for the transition temperatures.

Taking into account that there is a rather constant energy shift due to the difference between experimental and theoretical cohesive energies, and the fact that vibrational effects are not included in these calculations, we now examine the competition between a bcc and an fcc cell subject to the same temperature treatment for $\mathrm{Cu}_{60} \mathrm{Pd}_{40}$ alloys. Fig. 14 shows the energy vs. temperature curves for these cells, clearly showing that at high temperatures an fcc solid solution is favored, while at low temperatures, the ordered B2 phase is energetically favored. The figure suggests, however, that the transition from one regime (fcc, solid solution) to another (bcc, ordered B2 phase) takes place at unrealistic high temperatures $(3500 \mathrm{~K})$. While this simulation proves that there is a transition from a high temperature fcc solid solution to a low temperature bcc ordered phase, it does not provide an accurate tool for determining transition temperatures.

\section{CONCLUSIONS}

The extensive range of application described in this paper prove that it is possible to attack problems of different nature with a universal set of parameters, as long as the method breaks the dependence of the parameters on a specific type of atomic distribution. With the same level of accuracy, the BFS method was shown to provide the correct description of the bulk and surface behavior of single monatomic crystals ( $\mathrm{Cu}$ or $\mathrm{Pd}$ ), as well as their alloys. The only unavoidable 
dependence of the parameters is in terms of the crystal symmetry under consideration. However, once the crystal symmetry is determined (bcc, fcc), it is not necessary to change the parameters for specific applications. It is also shown that the use of first-principles methods provides an efficient tool for determining the BFS input parameters when there is no experimental source. In general, it is shown that the BFS method can be made independent altogether from experimental input, by basing the calculation of its input parameters solely in terms of first-principles calculations.

\section{ACKNOWLEDGMENTS}

Fruitful discussions with N. Bozzolo are gratefully acknowledged. The authors would like to thank C. Amador, B. Good, J. Ferrante, and A. Rodriguez for their contributions and comments. We also acknowledge the assistance of J. Khalil in the preparation of this manuscript. This work was supported by the HOTPC project at NASA Glenn Research Center.

\section{REFERENCES}

1. J. Hafner, Acta Mater. 48 (2000) 71.

2. J. K. Norskov, Phys. Rev. B 20 (1979) 446.

3. M. W. Finnis and J. E. Sinclair, Phil. Mag. A 50 (1984) 45.

4. G. Bozzolo, R. D. Noebe, J. Ferrante and C. Amador, J. Comput.-Aided Mater. Design 6 (1999) 1.

5. J. R. Smith, T. Perry, A. Banerjea, J. Ferrante and G. Bozzolo, Phys. Rev. B 44 (1991) 6444.

6. M. S. Daw and M. I. Baskes, Phys. Rev. Lett. 50 (1983) 1285.

7. J. H. Rose, J. R. Smith and J. Ferrante, J., Phys. Rev. B 28 (1983) 1835.

8. R. Hultgren, R. L. Orr, P. D. Anderson and K. K. Kelley, Selected Values of the Thermodynamic Properties of Binary Alloys (Wiley, New York, 1963) p. 779.

9. G. Bozzolo and J. Ferrante, J. Computer-Aided Mater. Design 2 (1995) 113.

10. G. Bozzolo, J. Ferrante, R. D. Noebe and C. Amador, Scripta Mater. 33 (1995) 1907.

11. G. Bozzolo, J. Ferrante, R. D. Noebe and C. Amador, Scripta Mater. 36 (1997) 813. 
12. G. Bozzolo, J. Ferrante, R. D. Noebe, B. Good, F. S. Honecy and P. Abel, Comp. Mat. Sci. 15 (1999) 169.

13. G. Bozzolo, R. D. Noebe, F. Honecy, Intermetallics 8 (2000) 7, and references therein.

14. J. R. Smith and A. Banerjea, Phys. Rev. B 37 (1988) 10411.

15. K. -M. Ho and K. P. Bohnen, Phys. Rev. B32 (1985) 3446.

16. C. L. Fu, S. Ohnishi, E. Wimmer and A. J. Freeman, Phys. Rev. Lett. 53 (1984) 675.

17. J. A. Appelbaum and D. R. Hamann, Solid State Comm. 27 (1978) 881.

18. K. -P. Bohnen and K. -M. Ho, Surf. Sci. 207 (1988) 105.

19. D. F. Ogeltree, M. A. van Hove and G. A. Somorjai, Surf. Sci., 173 (1986) 351.

20. P. J. Feibelman, Phys. Rev. B 46 (1992) 2532.

21. K. P. Bohnen, Th. Rodach and K. -M. Ho in: The Structure of Surfaces III, Eds. S. Y. Tong and M. A. Van Hove (SPringer, New York, 1991).

22. S. M. Foiles, M. I. Baskes and M. S. Daw, Phys. Rev. B 33 (1986) 7893.

23. T. Ning, Q. Yu and Y. Y. Ye, Surf. Sci. 206 (1988) L857.

24. D. Wolf, Surf. Sci. 226 (1990) 389.

25. J. G. Gay, J. R. Smith, R. Richter, F. J. Arlinghaus and R. H. Wagoner, J. Vac. Sci. Technol. A 2 (1984) 931; J. R. Smith, J. Ferrante, P. Vinet, J. G. Gay, R. Richter and J. H. Rose in: Chemistry and Physics of Fracture, Eds. R. H. Jones and R, M, Latanision (Nijhoff, Hingham, MA, 1987). 26. S. P. Chen and A. F. Voter, Surf. Sci. Lett. 244 (1991) L107.

27. M. Weinert, R. E. Watson, J. W. Davenport and G. W. Fernando, Phys Rev. B 39 (1989) 12585.

28. W. R. Tyson and W. A. Miller, Surf. Sci. 62 (1977) 267.

29. W. R. Tyson, J. Appl. Phys. 47 (1976) 459.

30. G. Bozzolo, J. Ferrante, and A. M. Rodriguez, J Comput.-Aided Mater. Design 1 (1993) 285. 31. H. L. Davis and J. R. Noonan, Surf. Sci. 126 (1983) 245.

32. D. M. Lind, F. B. Dunning, G. K. Walter, and H. L. Davis, Phys. Rev. B 35 (1987) 9037.

33. R. Mayer, C. Zhang, K. G. Lynn, W. E. Frieze, F. Jona and P. M. Marcus, Phys. Rev. B 35 (1987) 3102.

34. H. L. Davis and J. R. Noonan, J. Vac. Sci. Technol. 20 (1982) 842.

35. D. L. Adams, H. B. Nielsen, J. N. Andersen, I. Stensgaard, R. Feidenhans'l and J. E. Sorensen, Phys. Rev. Lett. 49 (1982) 669. 
36. D. L. Adams, H. B. Nielsen and J. N. Andersen, Surf. Sci. 128 (1983) 294.

37. H. L. Davis, J. R. Noonan and L. H. Jenkins, Surf. Sci. 83 (1979)559.

38. S. A. Lindgren, L. Wallden, J. Rundgren and P. Westrin, Phys. Rev. B 29 (1984) 576.

39. S. P. Tear, K. Roll and M. Prutton, J. Phys. C: Condens. Matter 14 (1981) 3297.

40. J. Quinn, Y. S. Li, D. Tian, H. Li, F. Jona and P. M. Marcus, Phys. Rev. B 42 (1990) 11348.

41. C. J. Barnes, M. Q. Ding, M. Lindroos, R. D. Diehl and D. A. King, Surf. Sci. 162 (1985) 59.

42. I. Stensgaard, R. Feidenhans'l and J. E. Sorensen, Surf. Sci. 128 (1983) 281.

43. M. Copel, T. Gustafsson, W. R. Graham and S. M. Yalisove, Phys. Rev. B 33 (1986) 810.

44. S. K. S. Ma, F. W. de Wette and G. P. Alldredge, Surf. Sci. 78 (1978) 598.

45. M. Methfessel, D. Hennig and M. Scheffler, Phys. Rev. B 46 (1992) 4816.

46. J. Burchardt, E. Lundgren, M. N. Nielsen, J. N. Andersen and D. L. Adams. Surf. Rev. Lett. 3 (1996) 1339.

47. R.J. Behm, K. Christmann, G. Ertl, M. A. Van Hove, P. A. Thiel and W. H. Weinberg, Surf. Sci. 88 (1979) L59.

48. M. E. Grillo, C. Stampfl and W. Berndt. Surf. Sci. 317 (1994) 84.

49. H. Ohtani, M. A. Van Hove and G. A. Somorjai. Surf. Sci. 187 (1987) 372.

50. O. L. Warren and P. A. Thiel, Phys. Rev. B 47 (1993) 10848.

51. R. D. Diehl, M. Lindroos, A. Kearsley, C. J. Barnes and D. A. King, J. Phys. C 18 (1985) 4069.

52. M. Skottke, R. J. Behm, G. Ertl, V. Penka and W. Moritz, J. Chem. Phys. 87 (6191)1987. 53. S. Durukanoglu and T. S. Rahman, Surf. Sci. 409 (1998) 395.

54. C. Cohen, A. L'Hoir, J. Moulin, D. Schmaus, M. Sotto, J. -L. Domange and J. -C. Boulliard, Surf. Sci. 339 (1995) 41.

55. M. Hirsimäkia, T. Pitkänen, M. Valdena, M. Lindroos and C. J. Barnes, Surf. Sci. 454-456 (2000) 6.

56. Th. Rodach, K. -P. Bohnen and K. M. Ho, Surf. Sci. 286 (1993) 66.

57. Ismail, S. Chandravakar, D. M. Zehner, Surf. Sci. 504 (2002) L201.

58. W. C. Johnson, J. M. Blakely, Interfacial Segregation, ASM, Metals Park, 1979.

59. R. D. Chaudhuri and Allen Miller, Surface Segregation Phenomena, Eds. P. A. Dowben and A. Miller (CRC Press, Boca Raton, 1990) 1. 
60. C. Gallis, B. Legrand, A. Saúl, G. Tréglia, P. Hecquet and B. Salanon, Surf. Sci. 352-354 (1996) 588.

61. E. Sagie, M. Polak, Surf. Sci. 459 (2000) 223.

62. P. Lejcek, O. Schneeweiss, Surf. Sci. 487 (2001) 210.

63. V. Blum, L. Hammer, W. Meier, K. Heinz, M. Schmid, E. Lundgren, P. Varga, Surf. Sci. 474 (2001) 81 .

64. E. C. Viljoen, J. du Plessis, Surf. Sci. 468 (2000) 27.

65. B. Moest, P. T. Wouda, A. W. Denier van der Gon, M. C. Langelaar, H. H. Brongersma, B. E. Nieuwenhuys, D. O. Boerma, Surf. Sci. 473 (2001) 159.

66. J. Creuze, F. Berthier, R. Tetot and B. Legrand, Surf. Sci. 491 (2001) 1.

67. D. Brown, P. D. Quiñn, D. P. Woodruff, T. C. Noakes and P. Bailey, Surf. Sci. 497 (2002) 1.

68. B. Aufray, H. Giordano, D. N. Seidman, Surf. Sci. 447 (2000) 180.

69. A. V. Ruban and H. L. Skriver, Comp. Mat. Sci. 15 (1999) 119, and references therein.

70. L. N. Gumen, E. P. Feldman, V. M. Yurchenko, T. N. Mel'nik, A. A. Krokhin, Surf. Sci. 445 (2000) 526.

71. Y. Teraoka, M. Komaki, Surf. Sci. 439 (1999) 1.

72. F. L. Williams and D. Nason, Surf. Sci. 45 (1974) 377.

73. T. S. King, Surface Segregation Phenomena, Eds. P. A. Dowben and A. Miller, (CRC Press, Boca Raton, 1990) 27.

74. S. Mukherjee and J. L. Moran-Lopez, Surf. Sci. 188 (1987) L742.

75. A. R. Miedema, Z. Metallk. 69 (1978) 455.

76. J. R. Chelikowsky, Surf. Sci. 139 (1984) L197.

77. M. A. Vasiliev, J. Phys. D: Appl. Phys. 30 (1997) 3037.

78. U. Bardi, Rep. Prog. Phys. 57 (1994) 939.

79. C. J. Barnes, E. AlShamaileh, T. Pitkänen, P. Kaukasoina and M. Lindroos, Surf. Sci. 492 (2001) 55 .

80. J. S. Tsay, T. Mangen and K. Wandelt, Thin Solid Films 397 (2001) 152.

81. J. Moon, J. Lowekamp, P. Wynblatt, S. Garoff and R. M. Suter, Surf. Sci. 488 (2001) 73.

82. R. Plass and G. L. Kellog, Surf. Sci. 470 (2000) 106.

83. S. Meza-Aguilar, O. Elmouhssine, H. Dreysse and C. Demangeat, Surf. Sci. 454-456 (2000) 900. 
84. P. W. Murray, I. Stengsgaard, E. Laegsgaard, F. Besenbacher, Phys. Rev. B 52 (1995) R14404. 85. P. W. Murray, I. Stengsgaard, E. Laegsgaard, F. Besenbacher, Surf. Sci. 365 (1996) 591.

86. T. D. Pope, M. Vos, H. T. Tang, K. Griffiths, I. V. Mitchell, P. R. Norton, W. Liu, Y. S. Li, A. R. Mitchell, Z. -J. Tian, J. E. Black, Surf. Sci. 337 (1995) 79.

87. Y. G. Shen, J. Yao, D. J. O'Connor, B. V. King, R. J. MacDonald, J. Phys.: Condens. Matter 8 (1996) 4903.

88. P. W. Murray, S. Thorshaug, I. Stensgaard, F. Besenbacher, E. Laegsgaard, A. V. Ruban, K. W. Jacobsen, G. Kopidakis, H. L. Skriver, Phys. Rev. B 55 (1997) 1380; R. A. Bennett, Spoulston, N. J. Price, J. P. Reilly, P. Stone, C. J. Barnes and M. Bowker, Surf. Sci. 433-435 (1999) 816. 89. E. Hahn, E. Kampshoff, A. Fricke, J.-P. Bucher, K. Kern, Surf. Sci. 319 (1994) 277. 90. J. P. Bucher, E. Hahn, P. Fernandez, C. Massobrio, K. Kern, Europhys. Lett. 27 (1994) 473. 91. J. E. Garces, H. O. Mosca, G. Bozzolo, Surf. Sci. 459 (2000) 365.

92. J. E. Garces, G. Bozzolo, P. Abel, H. O. Mosca, Appl. Surf. Sci. 167 (2000) 18.

93. J. O. Linde, Ann. Phys. Lpz. 15 (1932) 249.

94. J. M. Sanchez, F. Ducastelle, D. Gratias, Physica 128 A (1984) 334.

95. W. D. Connolly and A. R. Williams, Phys. Rev. B 27 (1983) 5169.

96. W. B. Pearson, Handbook of Lattice Spacings and Structure of Metals (Pergamon Press, New York, 1967).

97. A. M. Rodriguez, G. Bozzolo and J. Ferrante, Surf. Sci. 289 (1993) 100.

98. H. L. Davis, J. R. Noonan, Phys. Rev. Lett. 54 (1985) 566.

99. D. Sondericker, F. Jona, P.M.Marcus, Phys. Rev. B 33 (1986) 900.

100. Z. Q. Whang, Y. S. Li, C. K. C. Lok, J. Quinn, F. Jona and P. M. Marcus, Sol. State. Comm. 62 (1987) 181.

101. Y. G. Shen, D. J. O'Connor, K. Wandelt, Surf. Sci. 406 (1998) 23.

102. Y. G. Shen, D. J. O'Connor, K. Wandelt, Surf. Sci. 410 (1998) 1.

103. R. Kobistek, H. Schlosser, G. Bozzolo and J. Ferrante, Surf. Sci. $307 / 309$ (1994) 390.

104. W. E. Wallace and G. J. Ackland, Surf. Sci. Lett. 275 (1992) L685.

105. T. M. Buck, G. H. Wheatley and L. Marchut, Phys. Rev. Lett. 51 (1983) 43.

106. S. P. Chen, A. F. Voter and D. J. Srolovitz, Phys. Rev. Lett. 52 (1986) 1308.

107. S. M. Foiles, Surf. Sci. 191 (1987) 329.

108. J. Khalil, G. Bozzolo and R. D. Noebe, Comp. Mat. Sci. (2002) (in press). 
109. G. Bozzolo, H. Mosca, A. Wilson, R. D. Noebe, J. Garces, Met. Trans. B 33 (2002) 265.

110. G. Burns, Solid State Physics, Academic Press, New York, 1985.

111. O. K. Andersen, Phys. Rev. B 12 (1975) 3060.

112. P. Blaha P, K. Schwartz and J. Luitz, WIEN97, Vienna University of Technology. Improved and updated Unix version of the copyrighted WIEN code, P. Blaha, P. Schwartz, P. Sorantin and S.

B. Trickey, Comput. Phys. Commun. 59 (1990) 399. 


\section{TABLE CAPTIONS}

Table 1: ECT and BFS parameters. The constant $\mathrm{p}$ is $2 \mathrm{n}-2$, where $\mathrm{n}$ is the atomic principal quantum number, $l$ (in $\AA$ ) is a scaling length [7], the screening length $\lambda$ (in $\AA$ ) is defined as $2.81 l$ [5]. $E_{c}$ and $a_{e}$ are the cohesive energy (in $\mathrm{eV} / \mathrm{atom}$ ) and equilibrium lattice parameter (in $\AA$ ) of the pure crystal. The BFS parameters $\Delta_{C u P d}$ and $\Delta_{P d C u}$ were determined following the procedure in Ref. 9.

Table 2: Unrelaxed and relaxed surface energies of [hkl] $\mathrm{Cu}$ and $\mathrm{Pd}$ faces. In each case, we add the four ECT contributions to the total energy. Experimental and theoretical values of the surface energy of different $\mathrm{Cu}$ and $\mathrm{Pd}$ surfaces. The experimental results (which generally correspond to polycrystalline surfaces) are (in erg/ $\mathrm{cm}^{2}$ ) 1770 [28] and 1790 [29] for $\mathrm{Cu}$ and 2000 [28] for Pd.

Table 3: Interlayer relaxations for the low index faces of $\mathrm{Cu}$ and $\mathrm{Pd}$ as obtained by ECT and other methods.

Table 4: Multilayer relaxation of the (100), (110) and (111) faces of Cu. Experimental and theoretical results are included. For ECT, two different sets of results are presented: (a) One-layer, obtained by forcing the second layer to remain at its equilibrium position and (b) Two-layer, where the top two planes are allowed to relax. The experimental results include the experimental error, and the theoretical results include the variation in the predicted surface energies by $1 \%$ (see text), quoted as a 'theoretical' error.

Table 5: Perpendicular $(\Delta \mathrm{d})$ and parallel $(\Delta \mathrm{a})$ relaxations of $\mathrm{Cu}(210)$ and (311) surfaces expressed as percentages of the corresponding bulk spacings.

Table 6: Segregation trends in several binary systems. (-) means segregation of the impurity and $(+)$ means that segregation does not occur. See text for the definition and interpretation of the terms listed in the last three columns.

Table 7: Lattice spacings of the CuPd solid solution as a function of Pd concentration [96]. 
Table 8: Energies of formation [8] for the Cu-Pd solid solution at $1350 \mathrm{~K}$.

Table 9: Surface energies (in ergs $/ \mathrm{cm}^{2}$ ), planar relaxations (as percentages of the rigid interplanar spacing) of the top two layers of several low-index faces of the $L 1_{2}$ ordered structure. $\Delta_{\mathrm{ij}}(\mathrm{X})$ represents the relaxation between planes $i$ and $j$ of an atom of species $\mathrm{X}$ in layer $i$. The last column indicates the difference in position (in $\AA$ ) of an atom $A$ and an atom $B$ in the top layer.

Table 10: Cohesive energy (in eV/atom), equilibrium lattice parameter (in $\AA$ ) and scaling length (in $\AA$ ) for the fcc and bcc versions of $\mathrm{Cu}$ and $\mathrm{Pd}$, as obtained from first-principles calculations (LMTO [111], LAPW[112]) and from experiment (in the fcc case only).

Table 11: Energies of formation (in eV/atom) and equilibrium lattice parameter (in $\AA$ ) of the fcc and bcc ordered phases of $\mathrm{CuPd}$. For the latter, the experimental values are also included. 


\section{FIGURE CAPTIONS}

Fig. 1: Phase diagram of Cu-Pd [8]. The arrows indicate regions of the phase diagrams where the listed issues are readily subject to modeling, some of which include the BFS analysis presented in this work.

Fig. 2: Summary of the energy spectra $N$ Pd atoms $(N=1, \ldots, 8)$. The lowest energy state is indicated with a thick line. The thick dashed line indicates the first configuration that includes a Pd atom below the surface layer. The dotted lines indicate the first configuration that includes $\mathrm{Pd}$ atoms in the overlayer (the fact that such states do not appear for $\mathrm{N}=1,6$ and 8 is because configurations with $\mathrm{Pd}$ in the overlayer were not included in the catalogue of selected distributions $(\mathrm{N}=$ $6,8)$ or because they exceed the highest energy shown $(0.90 \mathrm{eV} / \mathrm{atom}))$. The lowest energy configuration for each value of $\mathrm{N}$ is also shown.

Fig. 3: 'Decay' chains for $M=0$ through $M=3$ states, starting with the seed state $b_{A}$, a Pd atom in a $\mathrm{Cu}$ surface site ( $\mathrm{M}$ denotes the number of $\mathrm{Pd}$ atoms). Subsequent configurations result from the addition of $\mathrm{Cu}$ atoms in the overlayer. $\triangle \mathrm{E}$ (in $\mathrm{eV} / \mathrm{atom}$ ) indicates the difference in energy between any given configuration and the initial state in the chain $\left(\mathrm{b}_{\mathrm{A}}\right)$. The inset displays one of the configurations shown in the diagram, where a Pd atom is in a surface site and three $\mathrm{Cu}$ atoms occupy adjoining sites in the overlayer. In this case, a larger value of the ECT parameter $\lambda$ was used $(\lambda=$ $10 \AA$ ) in order to account for the asymmetry of the surface.

Fig. 4: 'Decay' chain for $\mathrm{Cu}$ deposition on $\mathrm{Pd}(110)$ for increasing $\mathrm{Cu}$ coverage $(\mathrm{N}$ denotes the number of $\mathrm{Cu}$ atoms). Open circles represent $\mathrm{Cu}$ atoms in an overlayer site, and solid squares represent $\mathrm{Pd}$ steps. $\mathrm{Cu}$ atoms attached to the right of the step represent $\mathrm{Cu}$ 'fingers' growing in the [110] direction. Two consecutive fingers at more than nearest-neighbor distance are represented by slightly separated circles. Consecutive fingers in contact with each other represent 2D Cu islands attached to a Pd step edge. $\Delta \mathrm{E}$ is the energy of formation (in eV/atom) of the computational cell. A solid circle represents a $\mathrm{Cu}$ atom in a surface site, with the displaced $\mathrm{Pd}$ atom (in the overlayer) represented by a solid square. The inset displays one of the configurations shown in the diagram, where a 4-atom $\mathrm{Cu}$ atom cluster is attached to a $\mathrm{Pd}$ step. 
Fig. 5: Intermediate states during the cooling process of a 1008-atom cell representing a Cu alloy.

Fig. 6: Intermediate states during the cooling process of a 1008-atom cell representing a $\mathrm{Cu}_{75} \mathrm{Pd}_{25}$ alloy.

Fig. 7: Intermediate states during the cooling process of a 1008 -atom cell representing a $\mathrm{Cu}_{82} \mathrm{Pd}_{18}$ alloy.

Fig. 8: Intermediate states during the cooling process of a 1008-atom cell representing a $\mathrm{Cu}_{88} \mathrm{Pd}_{12}$ alioy.

Fig. 9: Energy as a function of temperature for $\mathrm{Cu}_{60} \mathrm{Pd}_{40}$ cells with LAPW (solid squares) and experimental (solid disks) parameters and $\mathrm{Cu}_{82} \mathrm{Pd}_{18}$ fcc cells with LAPW (open squares) and experimental (open circles) parameters.

Fig. 10: Energy vs. temperature for an fcc (solid squares) and a bcc (open circles) cell, both calculated using LAPW-based BFS parameters. 


\begin{tabular}{|c|c|c|c|c|c|c|}
\hline \multicolumn{7}{|c|}{ ECT parameters } \\
\hline & $\mathrm{p}$ & $l$ & $\alpha$ & $\lambda$ & $\mathrm{E}_{\mathrm{c}}$ & $a_{e}$ \\
\hline $\mathrm{Cu}$ & 6 & 0.272 & 2.935 & 0.765 & 3.50 & 3.615 \\
\hline Pd & 8 & 0.237 & 3.612 & 0.666 & 3.94 & 3.890 \\
\hline \multicolumn{7}{|c|}{ BFS parameters $\left(\AA^{-1}\right)$} \\
\hline & \multicolumn{3}{|c|}{$\Delta_{C u P d}=-0.0495$} & \multicolumn{2}{|c|}{-0.0431} & \\
\hline
\end{tabular}

Table 1 


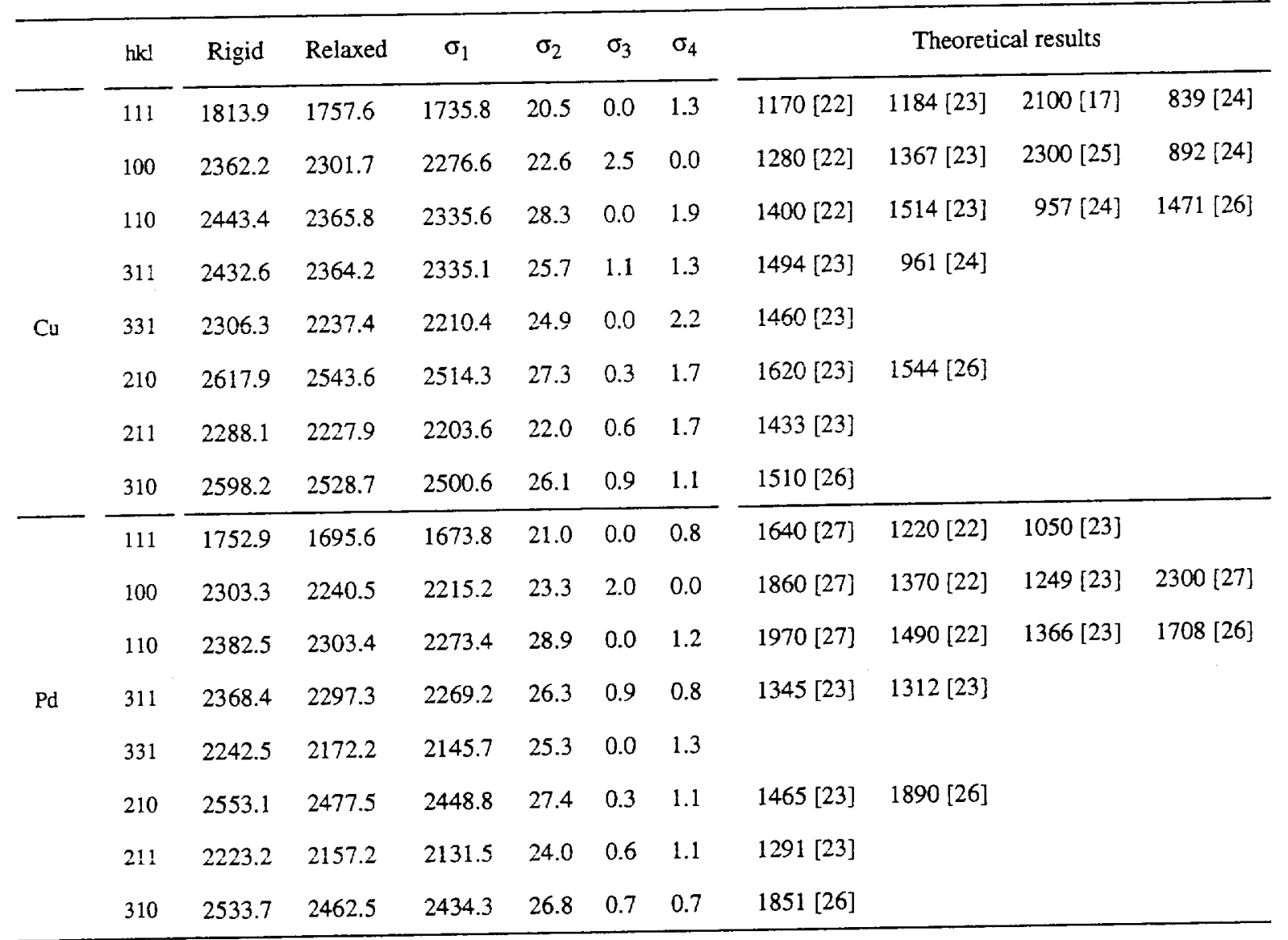

Table 2 


\begin{tabular}{|c|c|c|c|c|c|c|c|c|}
\hline \multirow[b]{2}{*}{$\mathrm{Cu}$} & \multicolumn{3}{|c|}{ Experiment } & \multicolumn{5}{|c|}{ Theory } \\
\hline & $\Delta_{12}$ & $\Delta_{23}$ & Ref. & $\Delta_{12}$ & $\Delta_{23}$ & $\Delta_{34}$ & $\Delta_{45}$ & Ref \\
\hline \multirow[t]{5}{*}{$\mathrm{Cu}(100)$} & -1.1 & +1.7 & [34] & -3.7 & +1.7 & & & ECT [30] \\
\hline & $-1.1+1-0.4$ & $+1.7+1-0.6$ & [31] & +1.0 & & & & {$[44]$} \\
\hline & $-1.0+/-0.4$ & $+2.0+1-0.8$ & [31] & -1.4 & -0.3 & & & {$[22]$} \\
\hline & -1.2 & +0.9 & {$[32]$} & -3.79 & -0.54 & +0.02 & 0.0 & [23] \\
\hline & -2.1 & +0.45 & [33] & & & & & \\
\hline \multirow[t]{8}{*}{$\mathrm{Cu}(110)$} & $-10.0+1-2.5$ & $0.0+/-2.5$ & [37] & -6.3 & +0.2 & & & ECT [30] \\
\hline & $-5.3+/-2.4$ & $+3.3+1-1.5$ & [35] & -4.9 & +0.23 & & & {$[22]$} \\
\hline & -8.5 & +2.3 & {$[35]$} & -8.73 & +1.53 & -1.20 & +0.43 & [23] \\
\hline & -7.9 & +2.4 & [31] & & & & & \\
\hline & -10.0 & +1.9 & {$[31]$} & & & & & \\
\hline & $-8.5+1-0.6$ & $+2.3+1-0.8$ & {$[36]$} & & & & & \\
\hline & $-7.5+/-1.5$ & $+2.5+1-1.5$ & {$[42]$} & & & & & \\
\hline & $-5.3+/-1.6$ & $+3.3+1-1.6$ & {$[43]$} & & & & & \\
\hline \multirow[t]{3}{*}{$\mathrm{Cu}(111)$} & $-0.3+/-1.0$ & & [39] & -3.1 & +1.6 & & & ECT [30] \\
\hline & $-0.7+1-0.5$ & & {$[38]$} & -1.4 & -0.04 & & & {$[22]$} \\
\hline & & & & -2.48 & -0.04 & 0.0 & 0.0 & [23] \\
\hline \multirow[t]{4}{*}{$\mathrm{Pd}(100)$} & +3.0 & -1.0 & {$[40]$} & -3.6 & +1.7 & & & ECT [30] \\
\hline & & & & -4.4 & -0.05 & & & [22] \\
\hline & & & & -4.94 & +0.17 & -0.05 & -0.08 & [23] \\
\hline & & & & -0.6 & & & & [45] \\
\hline \multirow[t]{4}{*}{$\operatorname{Pd}(110)$} & $4.0+1-1.5$ & $+1.5+/-1.5$ & {$[50]$} & -5.1 & +0.2 & & & ECT [30] \\
\hline & $-6.0+1-2.0$ & $+1.0+1-2.0$ & {$[41]$} & -11.3 & +1.2 & & & [22] \\
\hline & $-5.7+1-2.0$ & $+0.5+/-2.0$ & {$[51]$} & -11.2 & +2.49 & -1.18 & +0.40 & [23] \\
\hline & $-5.1+/-1.5$ & $+2.9+1-1.5$ & [52] & -5.3 & & & & [45] \\
\hline \multirow[t]{4}{*}{$\operatorname{Pd}(111)$} & & & & -2.4 & +1.3 & & & ECT [30] \\
\hline & & & & -3.2 & +0.27 & & & [22] \\
\hline & & & & -3.17 & +0.27 & -0.08 & -0.06 & [23] \\
\hline & & & & -0.1 & & & & {$[45]$} \\
\hline
\end{tabular}

Table 3 


\begin{tabular}{|c|c|c|c|c|c|c|}
\hline \multirow[b]{2}{*}{ Face } & \multicolumn{3}{|c|}{ Experiment } & \multirow{2}{*}{$\frac{\mathrm{ECT} \text { (One-layer) }}{\Delta \mathrm{d}_{12}}$} & \multicolumn{2}{|c|}{ ECT(Two-layers) } \\
\hline & $\Delta \mathrm{d}_{12}$ & $\Delta \mathrm{d}_{23}$ & Ref & & $\Delta \mathrm{d}_{12}$ & $\Delta \mathrm{d}_{23}$ \\
\hline$(100)$ & -2.1 & +0.45 & {$[33]$} & $-3.52+/-1.74$ & $-3.81+1-1.70$ & $+2.47+1-0.86$ \\
\hline$(110)$ & $-7.5+/-1.5$ & $+2.5+l-1.5$ & {$[42]$} & $-6.31+1-2.46$ & $-6.51+/-3.83$ & $+0.29+1.2 .44$ \\
\hline (111) & $-0.7+/-0.5$ & & {$[38]$} & $-2.88+1-1.30$ & $-3.10+1-1.25$ & $+2.12+/-0.63$ \\
\hline
\end{tabular}

Table 4 


\begin{tabular}{cccc}
\hline & $\begin{array}{c}\mathrm{Cu}(210) \\
{[\mathrm{ECT}]}\end{array}$ & $\begin{array}{c}\mathrm{Cu}(210) \\
{[57]}\end{array}$ & $\begin{array}{c}\mathrm{Cu}(311) \\
{[\mathrm{ECT}]}\end{array}$ \\
\hline$\Delta \mathrm{d}_{12}$ & $-4.48+/-5.07$ & $--11.12+/-2.0$ & $-5.54+/-3.27$ \\
$\Delta \mathrm{d}_{23}$ & $-4.91+/-4.17$ & $-5.68+/-2.3$ & $-0.81+/-2.99$ \\
$\Delta \mathrm{d}_{34}$ & $+0.96+/-4.38$ & $+3.83+/-2.5$ & $-1.75+/-4.33$ \\
$\Delta \mathrm{d}_{45}$ & $-2.03+/-5.80$ & $+0.06+/-3.0$ & $+4.32+/-5.05$ \\
$\Delta \mathrm{d}_{56}$ & $+3.20+/-6.80$ & $-0.66+/-3.5$ & $-1.60+/-5.02$ \\
\hline & & $\mathrm{Cu}(210)$ & $\mathrm{Cu}(311)$ \\
\hline$\Delta \mathrm{a}_{12}$ & $0.00+/-2.43$ & $-1.83+/-3.0$ & $+0.55+/-2.79$ \\
$\Delta \mathrm{a}_{23}$ & $+0.05+/-2.55$ & $-2.51+/-3.2$ & $+0.50+/-3.12$ \\
$\Delta \mathrm{a}_{34}$ & $+0.48+/-2.82$ & $+1.68+/-3.5$ & $-0.54+/-4.00$ \\
$\Delta \mathrm{a}_{45}$ & $+0.11+/-3.49$ & $-0.48+/-3.7$ & $+0.50+/-5.08$ \\
$\Delta \mathrm{a}_{56}$ & $-0.50+/-4.31$ & $+0.06+/-4.0$ & $-0.30+/-4.73$ \\
\hline
\end{tabular}

Table 5 


\begin{tabular}{|c|c|c|c|c|c|c|}
\hline Host & Impurity & Experiment & BFS & $\Delta S_{S}^{(0)}$ & $\varepsilon_{B_{0}}^{S}-\varepsilon_{B_{b}}^{S}$ & $\varepsilon_{B_{0}}^{S}-\varepsilon_{A_{0}}^{S}$ \\
\hline $\mathrm{Cu}$ & $\mathrm{Pd}$ & & - & - & + & - \\
\hline $\mathrm{Pd}$ & $\mathrm{Cu}$ & & + & + & + & + \\
\hline $\mathrm{Cu}$ & $\mathrm{Ni}$ & + & + & + & + & + \\
\hline $\mathrm{Ni}$ & $\mathrm{Cu}$ & - & - & - & + & - \\
\hline $\mathrm{Ag}$ & $\mathrm{Cu}$ & + & + & + & + & + \\
\hline $\mathrm{Cu}$ & $\mathrm{Ag}$ & - & - & - & + & - \\
\hline $\mathrm{Ag}$ & $\mathrm{Pd}$ & & + & + & + & + \\
\hline $\mathrm{Pd}$ & $\mathrm{Ag}$ & - & - & - & + & - \\
\hline $\mathrm{Au}$ & $\mathrm{Cu}$ & + & + & + & + & + \\
\hline $\mathrm{Cu}$ & $\mathrm{Au}$ & - & - & - & - & - \\
\hline $\mathrm{Au}$ & $\mathrm{Pd}$ & + & + & + & + & + \\
\hline $\mathrm{Pd}$ & $\mathrm{Au}$ & - & - & - & + & - \\
\hline $\mathrm{Ni}$ & $\mathrm{Pd}$ & - & - & - & - & - \\
\hline Pd & $\mathrm{Ni}$ & + & + & + & + & + \\
\hline
\end{tabular}

Table 6 


\begin{tabular}{ccc}
\hline at.\% Pd & a & BFS $[\AA]$ \\
\hline 0 & 3.615 & 3.615 \\
25.0 & 3.694 & 3.705 \\
51.9 & 3.774 & $3.774(50$ at.\% Pd) \\
78.4 & 3.839 & $3.834(75$ at.\% Pd) \\
100 & 3.892 & 3.89 \\
\hline
\end{tabular}

Table 7 


\begin{tabular}{ccc}
\hline $\mathrm{x}_{\mathrm{Cu}}$ & $\Delta H(\mathrm{Exp})$ & $\Delta H(\mathrm{BFS})$ \\
\hline 0.1 & -0.03920 & -0.0395 \\
0.2 & -0.07216 & -0.0719 \\
0.3 & -0.09141 & -0.0842 \\
0.4 & -0.10503 & -0.0921 \\
0.5 & -0.11088 & -0.1010 \\
0.6 & -0.11365 & -0.1052 \\
0.7 & -0.10581 & -0.0871 \\
0.8 & -0.07935 & -0.0793 \\
0.9 & -0.04358 & -0.0324 \\
\hline
\end{tabular}

Table 8 


\begin{tabular}{cccccccc}
\hline $\mathrm{A}_{3} \mathrm{~B}$ & Face & $\sigma$ & $\Delta_{12}(\mathrm{~A})$ & $\Delta_{12}(\mathrm{~B})$ & $\Delta_{23}(\mathrm{~A})$ & $\Delta_{23}(\mathrm{~B})$ & Ripple \\
\hline \multirow{6}{*}{$\mathrm{Cu} \mathrm{u}_{3} \mathrm{Pd}$} & $(100) 1: 1$ & 2341.92 & $-5.58+/-0.51$ & $-6.83+/-0.62$ & $+0.43+/-0.36$ & & 0.02328 \\
& $(100) 1: 0$ & 2259.46 & $-7.17+/-0.51$ & & $-5.89+/-0.75$ & $+1.32+/-0.62$ & \\
& $(110) 1: 1$ & 2520.31 & $-8.21+/-1.01$ & $-12.02+/-0.84$ & $+2.30+/-1.38$ & & 0.04985 \\
& $(110) 1: 0$ & 2517.05 & $-9.82+/-0.70$ & & $+1.34+/-2.28$ & $-8.39+/-2.10$ & \\
& $(111) 3: 1$ & 1793.00 & $+3.90+/-0.33$ & $-0.83+/-0.41$ & $+7.38+/-0.28$ & $+11.27+/-0.54$ & 0.10104 \\
\hline
\end{tabular}

Table 9 


\begin{tabular}{|c|c|c|c|c|}
\hline Element & Method & $E c$ & $a_{e}$ & $l$ \\
\hline \multirow{3}{*}{ Cü-f́cc } & Experiment & 3.50 & 3.615 & 0.2720 \\
\hline & LMTO & 4.437 & 3.5522 & 0.2705 \\
\hline & LAPW & 3.6683 & 3.6326 & 0.2786 \\
\hline \multirow[t]{3}{*}{$\mathrm{Cu}-\mathrm{bcc}$} & LMTO & 4.438 & 2.8225 & 0.2710 \\
\hline & LAPW & 3.6279 & 2.8891 & 0.2807 \\
\hline & Experiment & 3.94 & 3.89 & 0.2370 \\
\hline \multirow{2}{*}{ Pd-fcc } & LMTO & 4.788 & 3.9055 & 0.2487 \\
\hline & LAPW & 3.8033 & 3.9441 & 0.2474 \\
\hline \multirow[t]{2}{*}{ Pd-bcc } & LMTO & 4.786 . & 3.1069 & 0.2494 \\
\hline & LAPW & 3.7476 & 3.1427 & 0.2516 \\
\hline
\end{tabular}

Table 10 


\begin{tabular}{|c|c|c|c|}
\hline Alloy & Method & $\Delta H_{0}$ & $a_{0}$ \\
\hline \multirow{2}{*}{$\begin{array}{l}\text { Cu-Pd } \\
\left(\mathrm{L} 1_{0}\right)\end{array}$} & LMTO & -0.0875 & 3.749 \\
\hline & LAPW & -0.0944 & 3.8107 \\
\hline \multirow{3}{*}{$\begin{array}{l}\mathrm{Cu}-\mathrm{Pd} \\
\text { (B2) }\end{array}$} & Experiment & -0.142 & 2.958 \\
\hline & LMTO & -0.146 & 2.9716 \\
\hline & LAPW & -0.165 & 3.0199 \\
\hline
\end{tabular}

Table 11 


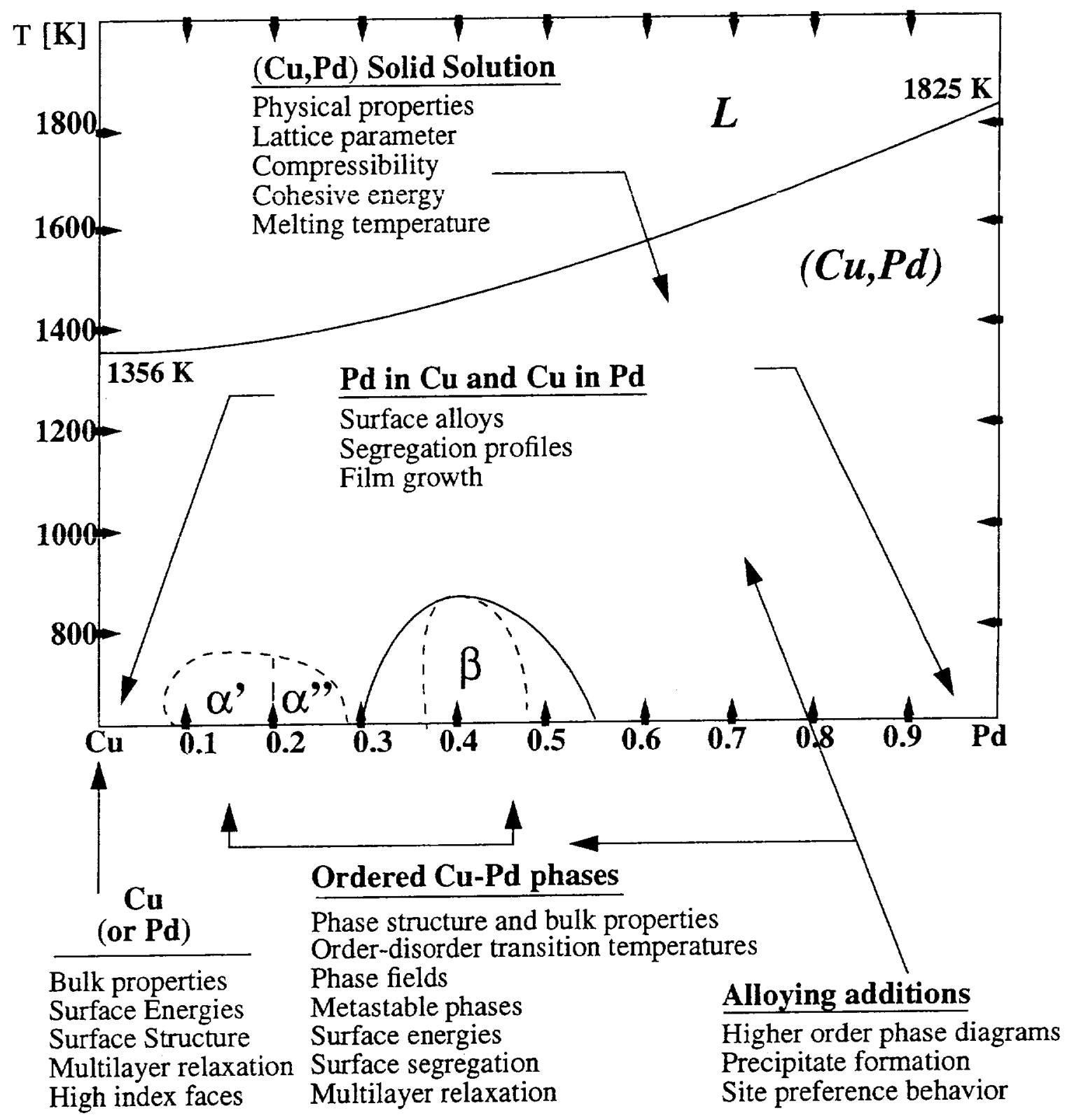

Fig. 1 


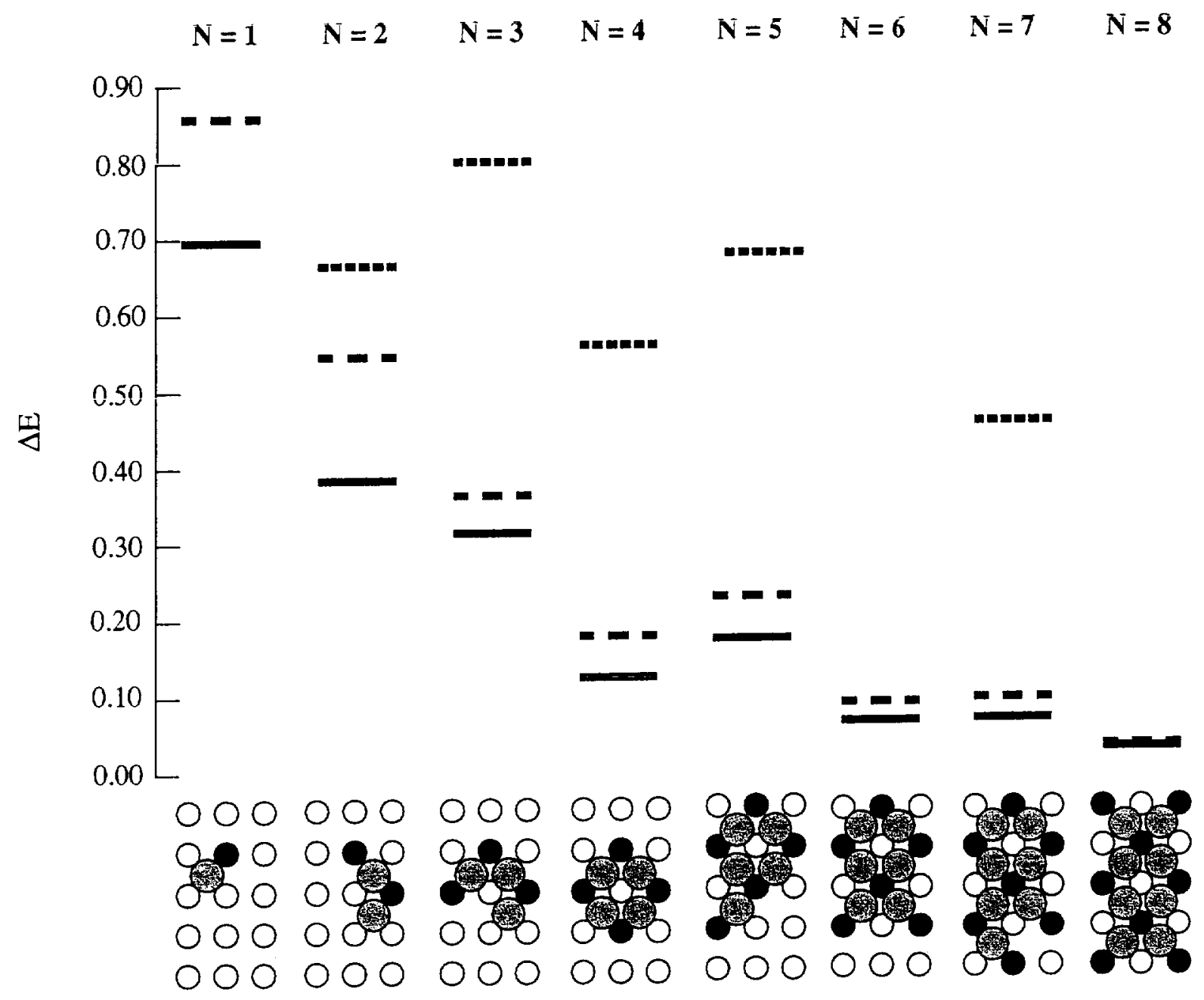

Fig. 2 


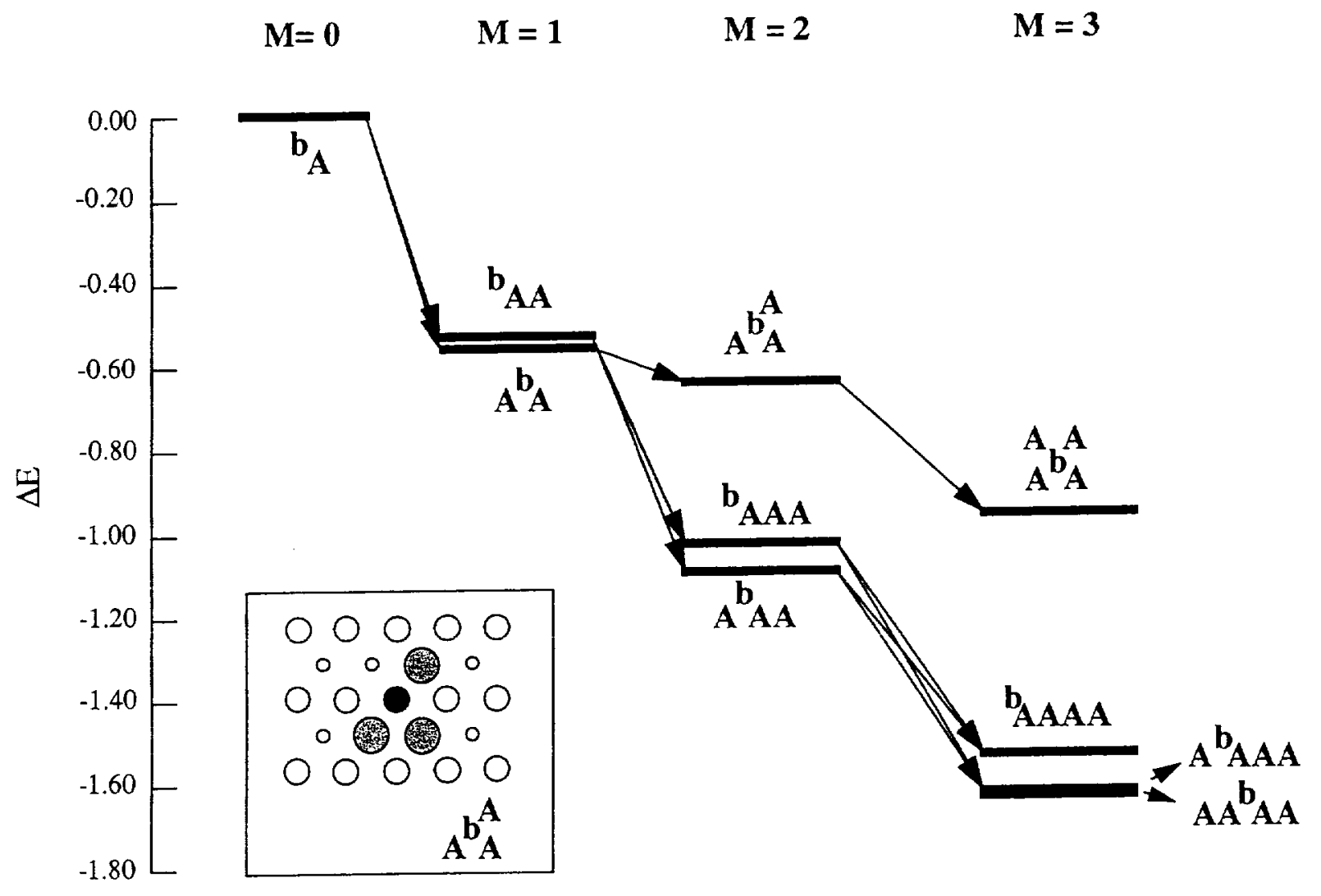

Fig. 3 


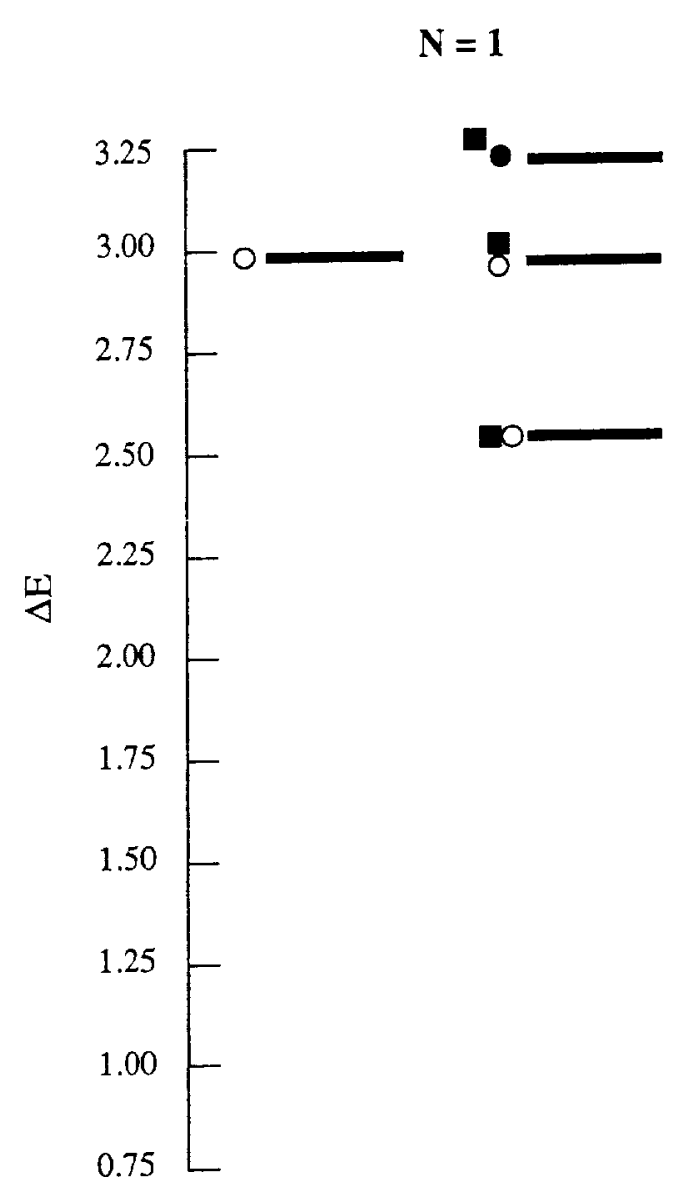

$$
\mathbf{N}=\mathbf{2}
$$$$
\mathbf{N}=\mathbf{3}
$$$$
\mathrm{N}=4
$$
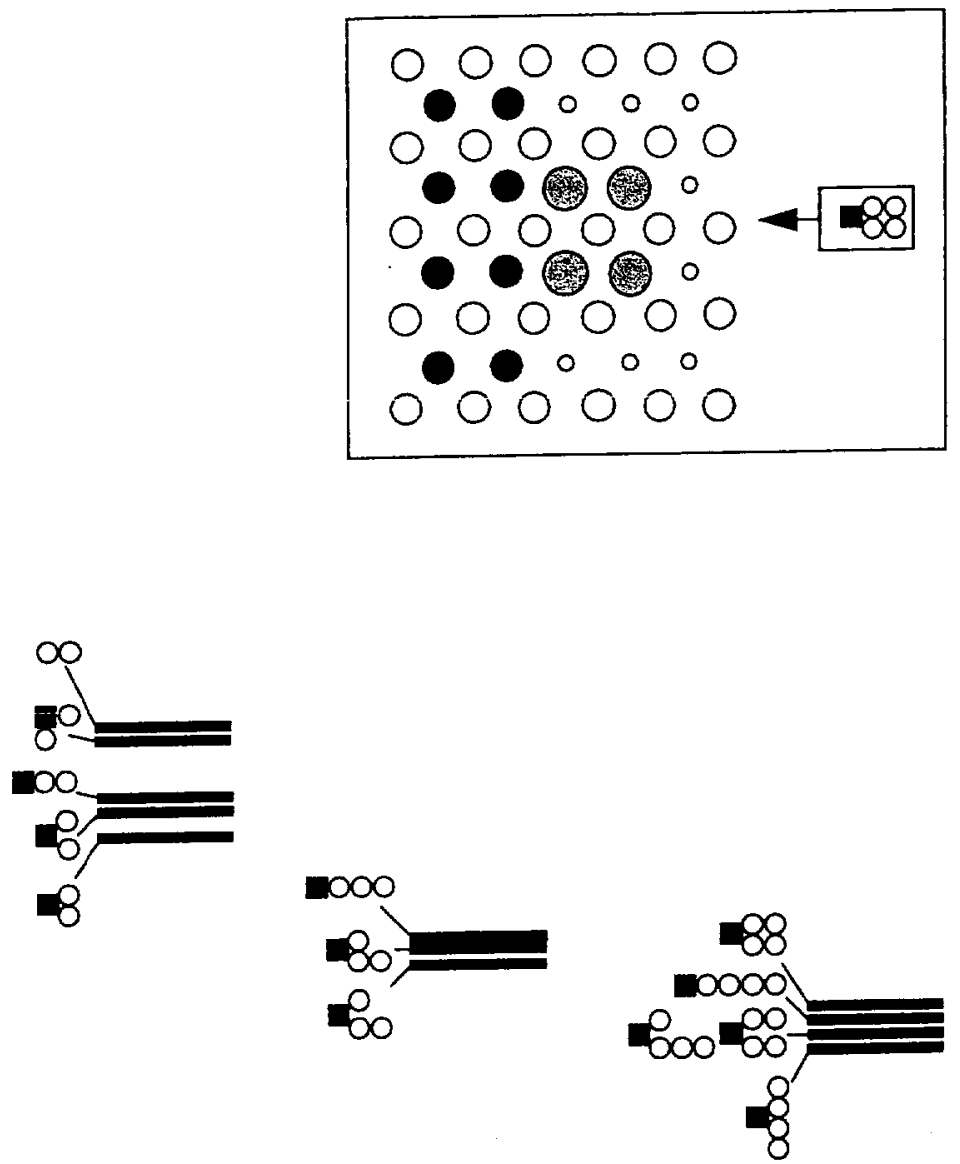

Fig. 4 


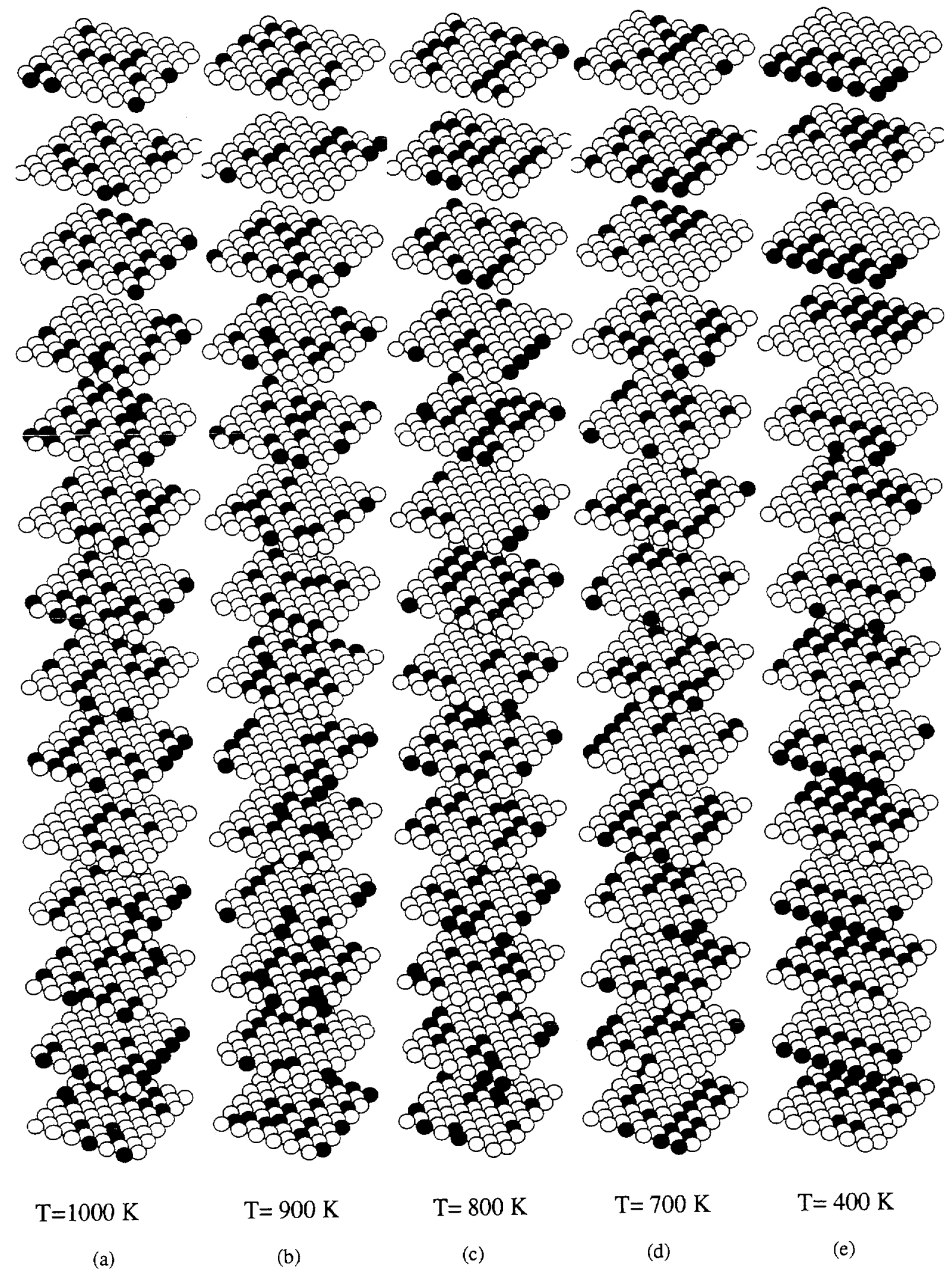

Fig. 5 


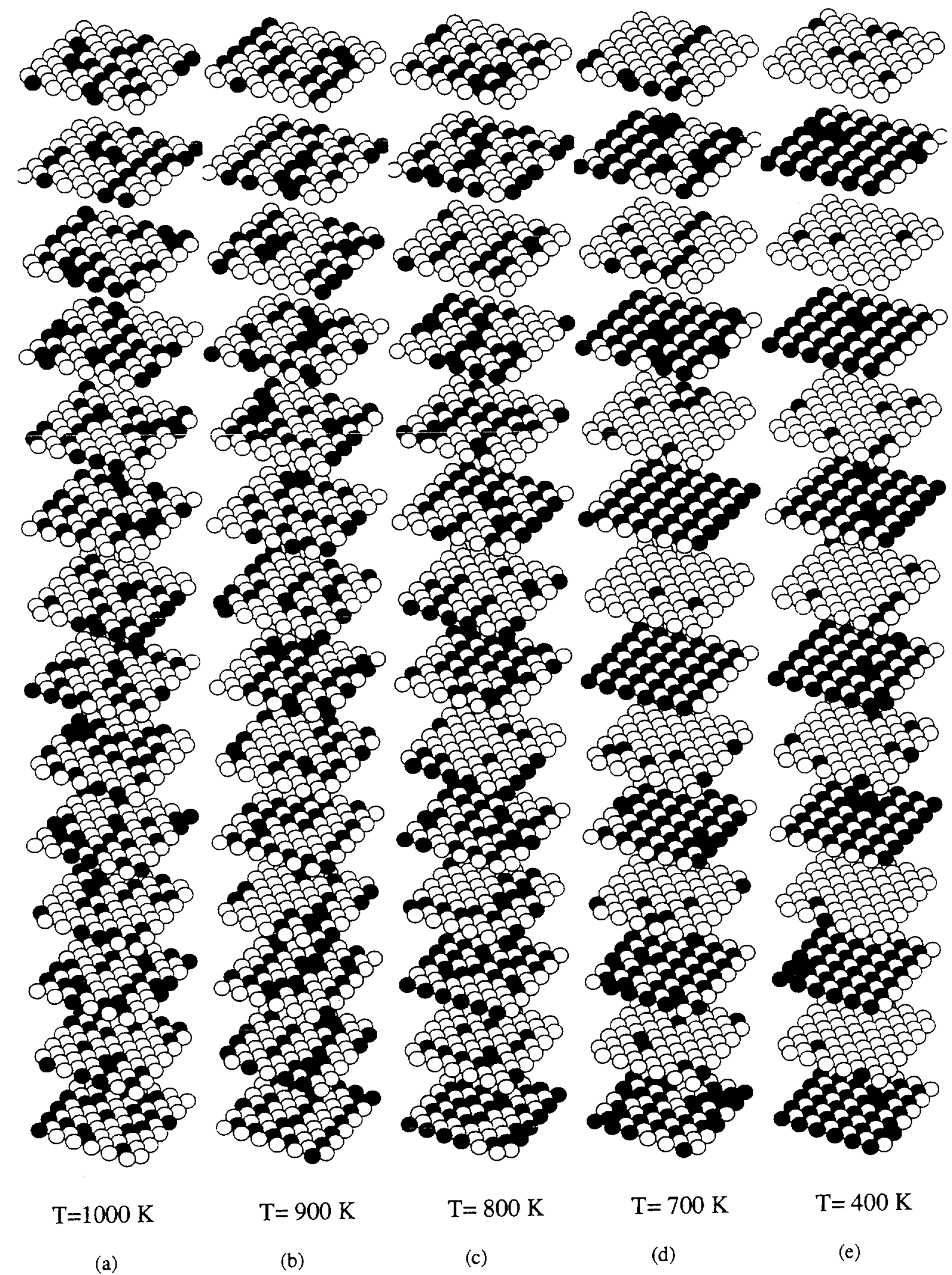

Fig. 6 


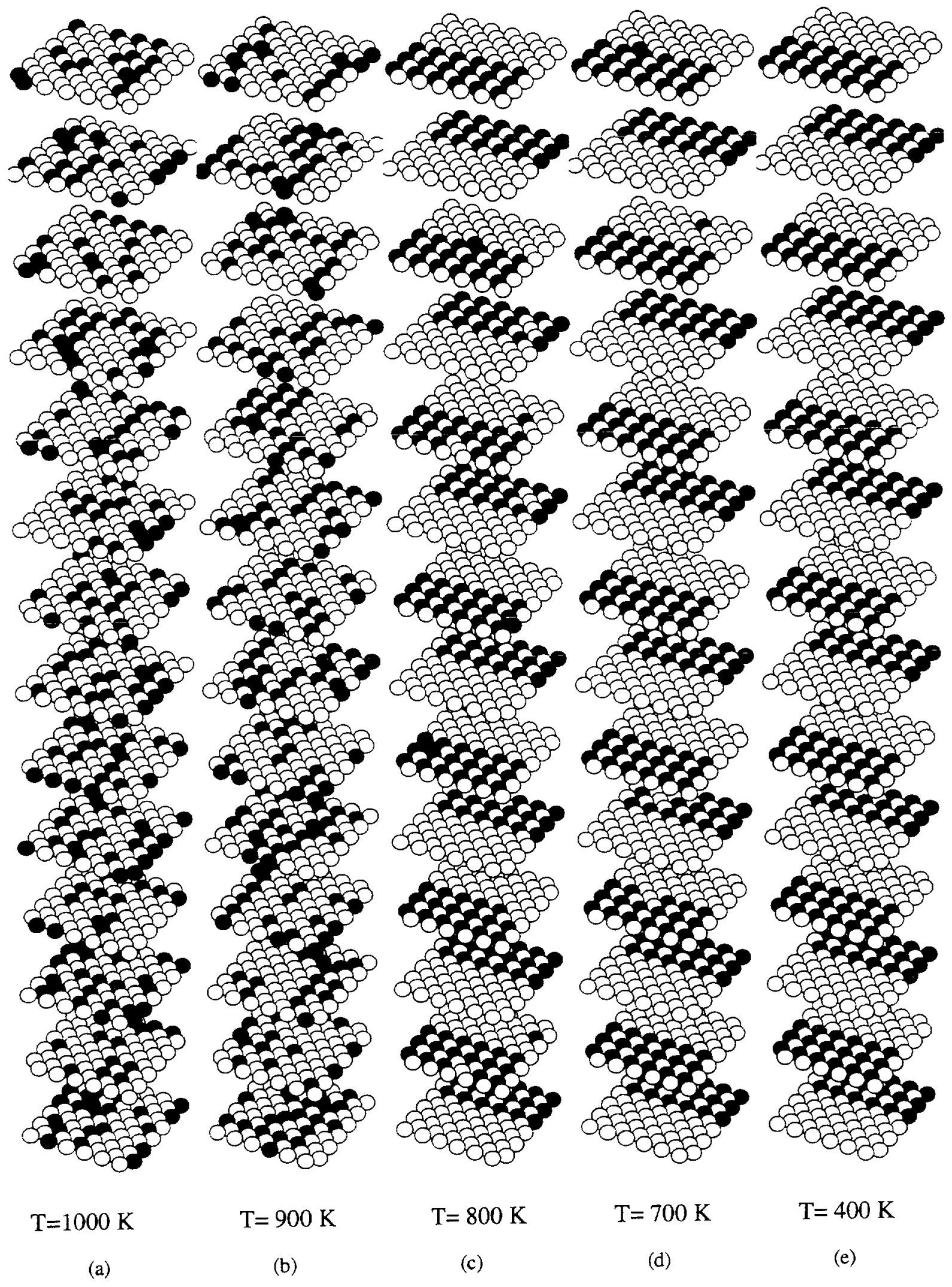

Fig. 7 


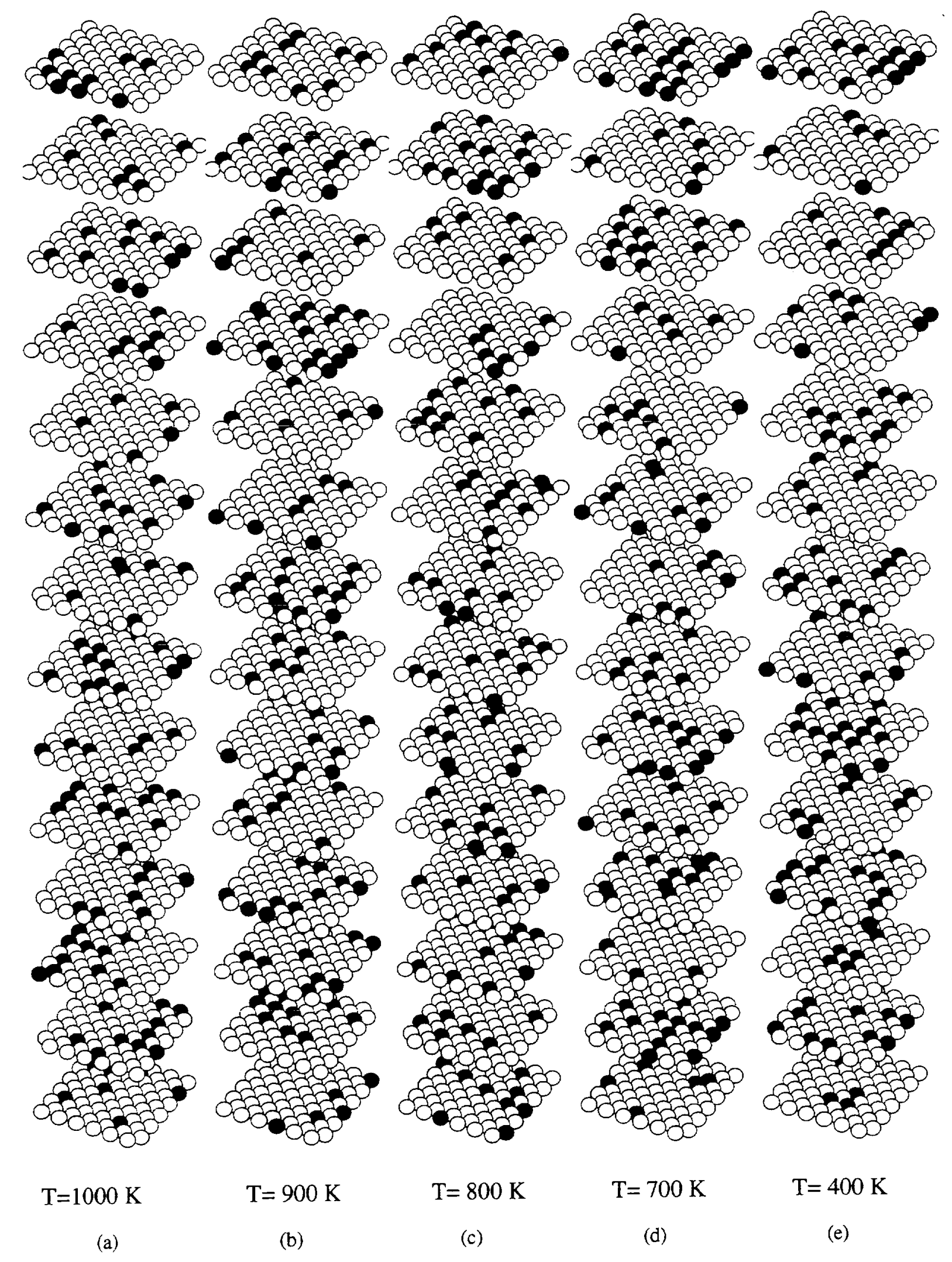

Fig. 8 


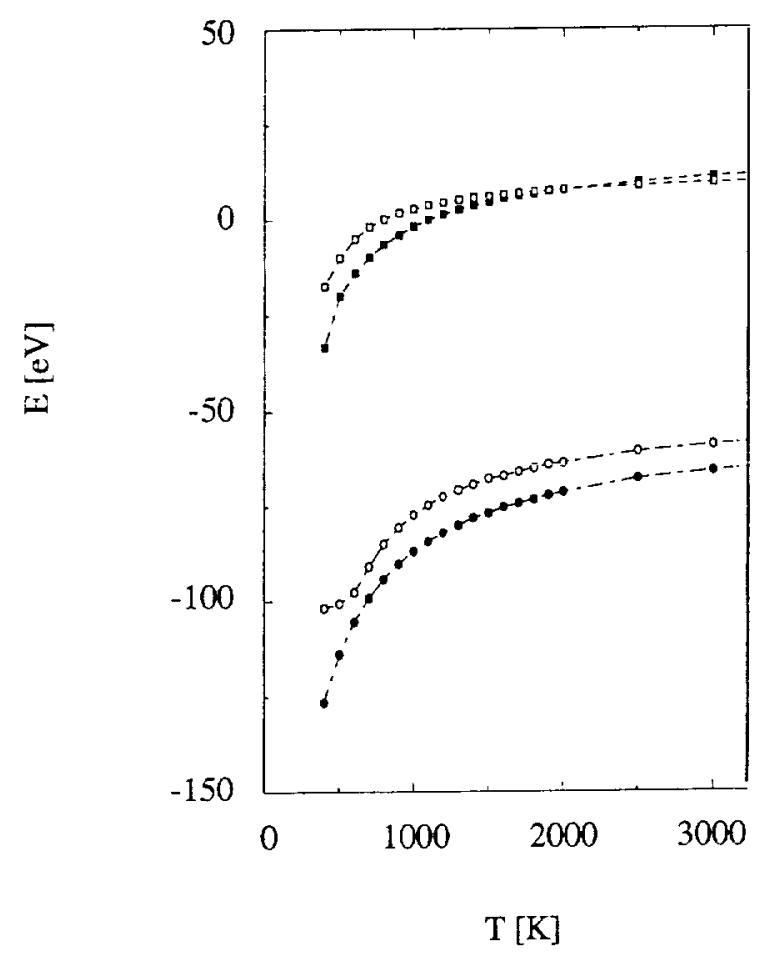

Fig. 9 


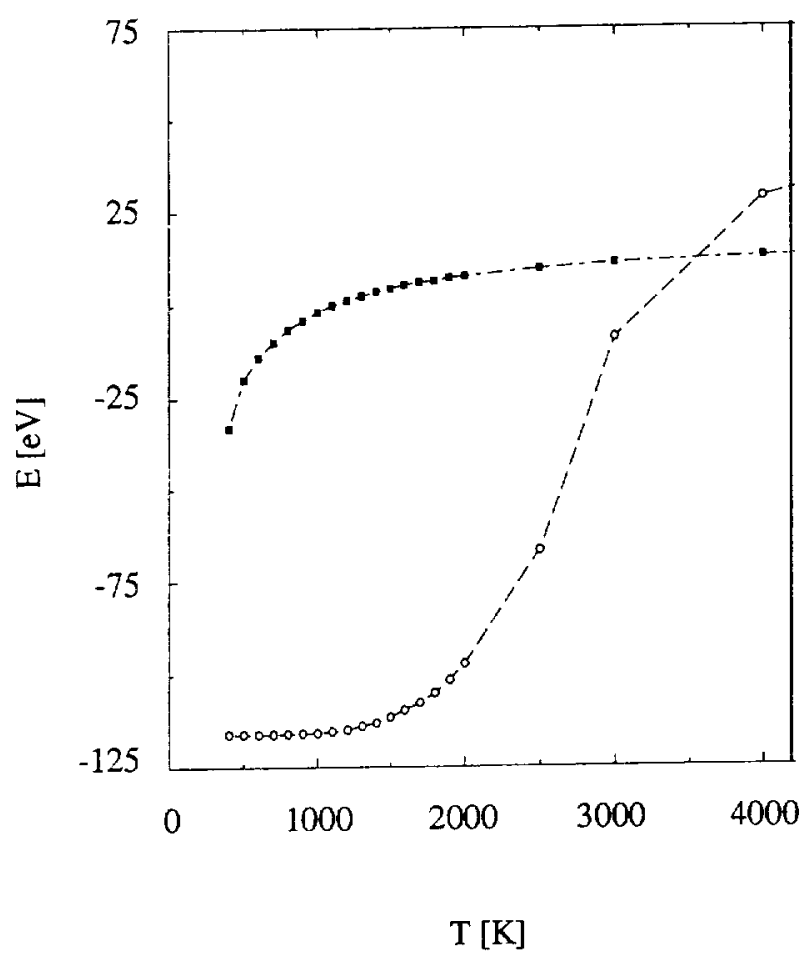

Fig. 10 